\title{
Separatrix regions of magnetic reconnection at the magnetopause
}

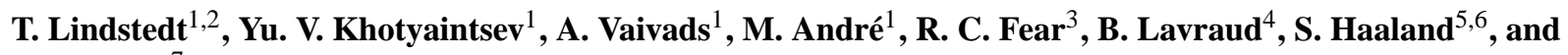 \\ C. J. Owen ${ }^{7}$ \\ ${ }^{1}$ Swedish Institute of Space Physics, P.O. Box 537, 75121 Uppsala, Sweden \\ ${ }^{2}$ Department of Physics and Astronomy, Space and Plasma Physics, P.O. Box 515, 75120 Uppsala, Sweden \\ ${ }^{3}$ Department of Physics and Astronomy, University of Leicester, Leicester LE1 7RH, UK \\ ${ }^{4}$ Centre d'Etude Spatiale des Rayonnements, CNRS, Toulouse, France \\ ${ }^{5}$ Department of Physics and Technology, University of Bergen, Bergen, Norway \\ ${ }^{6}$ Max-Planck-Institut für Extraterrestrische Physik, Garching, Germany \\ ${ }^{7}$ Mullard Space Science Laboratory, University College London, Dorking, UK
}

Received: 3 November 2008 - Revised: 7 October 2009 - Accepted: 7 October 2009 - Published: 26 October 2009

\begin{abstract}
Using data from the four Cluster spacecraft we study the separatrix regions of magnetic reconnection sites at the dayside magnetopause under conditions when reconnection is occurring in the magnetopause current layer which separates magnetosheath plasma from the hot magnetospheric plasma sheet. We define the separatrix region as the region between the separatrix - the first field line opened by reconnection - and the reconnection jet (outflow region). We analyze eight separatrix region crossings on the magnetospheric side of the magnetopause and present detailed data for two of the events. We show that characteristic widths of the separatrix regions are of the order of ten ion inertial lengths at the magnetopause. Narrow separatrix regions with widths comparable to a few ion inertial lengths are rare. We show that inside the separatrix region there is a density cavity which sometimes has complex internal structure with multiple density dips. Strong electric fields exist inside the separatrix regions and the electric potential drop across the regions can be up to several $\mathrm{kV}$. On the magnetosheath side of the region there is a density gradient with strong field aligned currents. The observed strong electric fields and currents inside the separatrix region can be important for a local energization of ions and electrons, particularly of ionospheric origin, as well as for magnetosphere-ionosphere coupling.
\end{abstract}

Keywords. Magnetospheric physics (Magnetopause, cusp, and boundary layers) - Space plasma physics (Discontinuities; Magnetic reconnection)

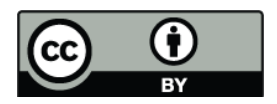

Correspondence to: $\mathrm{T}$. Lindstedt (toli@irfu.se)

\section{Introduction}

Magnetic reconnection is an important process that occurs in different plasma environments: in the Earth's magnetosphere, at the Sun, in astrophysical plasmas as well as in laboratory plasmas (Biskamp, 2000). At the Earth's magnetopause, magnetic reconnection is the dominant process responsible for the entry of solar wind plasma into the magnetosphere, as well as for the transfer of energy across the magnetopause.

Reconnection geometry can be divided into two main regions: these are the inflow region where plasma is on a large scale drifting towards the current sheet, and the outflow region where accelerated plasma jets away from the X-line. The regions between outflow and inflow regions can have complicated structures that depends on the properties of the reconnecting plasmas. These regions can be described in different ways. In the MHD description of steady reconnection of two similar plasmas, outflow and inflow regions are separated by a pair of slow shocks originating in the diffusion region (Petschek, 1964). In the case of asymmetric reconnection, as at the magnetopause, the MHD description predicts an Alfvén wave (rotational discontinuity) on the magnetosheath side and a slow expansion fan on the magnetospheric side (Levy et al., 1964). Depending on the asymmetry the structure of discontinuities can be different (Lin and Lee, 1994). The simplified MHD description of discontinuities has been successful in explaining low altitude observations of ion and electron signatures (Lockwood et al., 1996). More complicated MHD discontinuities develop when plasmas are different on either side of the current sheet and/or the reconnection is time varying (Semenov et al., 1992; Biernat et al., 1998).

Published by Copernicus Publications on behalf of the European Geosciences Union. 


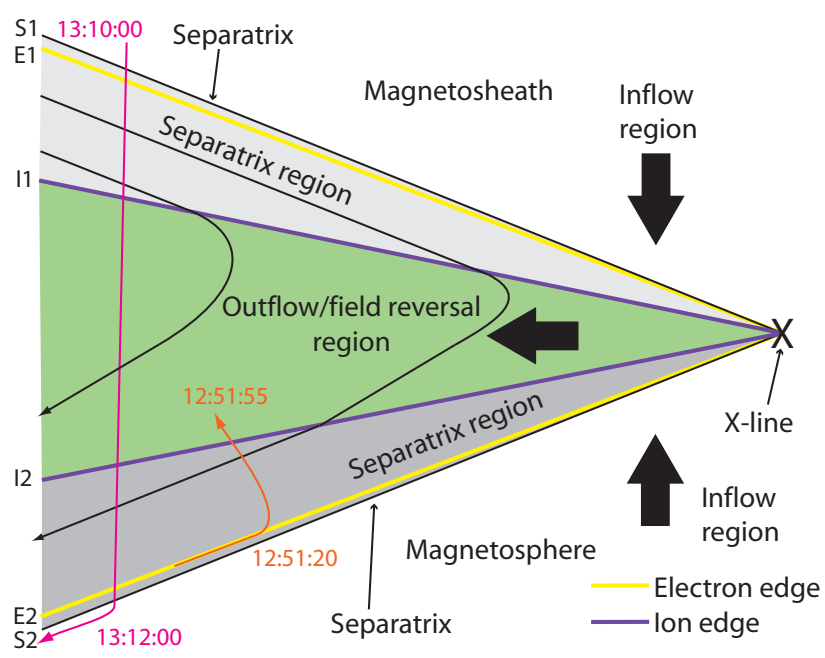

Fig. 1. Simplified sketch of a reconnection layer formed by an $\mathrm{X}$-line at the magnetopause. The separatrices are the magnetic field lines connected to the reconnection X-line. Just inside the separatrix are the electron edges in yellow. The purple lines illustrate the ion edges that are closer together than the electron edges due to the time-of-flight effect. Between the separatrices and ion edges are the separatrix regions in grey. Between the ion edges is the outflow region. Spacecraft orbits for the two events that are discussed in detail are shown by orange (12:51 UT) and pink (13:11 UT) lines. This figure is similar to Fig. 5 in Gosling et al. (1990).

Detailed experimental studies reveal that it is usually difficult to identify fluid boundaries in the data, such as slow shocks. Instead, it is useful to introduce the separatrix region (see Fig. 1). The separatrix region is between the separatrix, the first field line opened by reconnection, and the reconnection jet (outflow/field reversal region, Fig. 1). To identify the separatrix region kinetic particle signatures are important (Gosling et al., 1990; Vaivads et al., 2006; Khotyaintsev et al., 2006). The separatrix regions are at one side bounded by the separatrices - the magnetic field lines connected to the reconnection $\mathrm{X}$-line. The separatrices are located very close to the electron edges as electrons propagate relatively fast along the magnetic field. The magnetospheric electron edge (E2) is where (going from the magnetosphere to the magnetosheath) the first electrons originating in the magnetosheath are observed. Similarly, the magnetosheath electron edge (E1) is located where (going from the magnetosheath to the magnetosphere) the first magnetospheric electrons are observed. The separatrix regions contain a mixture of magnetospheric and magnetosheath electrons but not of ions. The ion edges are defined in a similar way (see also Gosling et al., 1990). In some studies a difference is made between the ion edge and the fluid boundary of the reconnection jet (Bogdanova et al., 2006). In particular, this is possible to do at lower altitudes. However, similar to Gosling et al. (1990), we do not make such a distinction because at the magne- topause it can be difficult to separate these boundaries. Thus, at the magnetospheric side the ion edge approximately coincides with the boundary of the reconnection jet (outflow/field reversal region in Fig. 1). Most of the magnetic field rotation from the magnetosheath to magnetospheric orientation happens inside the outflow/field reversal region bounded by the ion edges.

The separatrix region on the magnetospheric side of dayside magnetopause has been extensively studied at low altitudes (Lockwood et al., 1994, 1996; Sandholt et al., 1998, 2002) and mid (5-7 $R_{E}$ ) altitudes (Topliss et al., 2001; Bogdanova et al., 2004, 2006), as well as close to the magnetopause (Gosling et al., 1990; Khotyaintsev et al., 2006; Retinò et al., 2006). In some studies the separatrix region is referred to as the electron edge of the low latitude boundary layer (e.g. Bogdanova et al., 2006). On the basis of 8 events Bogdanova et al. (2004) discuss plasma and wave signatures characteristic for the separatrix regions (ULF waves, electron beams and outflow of ionospheric ions). Topliss et al. (2001) and Bogdanova et al. (2006) presented large statistical studies of this region. They found that the region can be up to $2^{\circ}$ ILAT thick. The median thickness was estimated to $\sim 0.2^{\circ}$ ILAT corresponding to roughly about $600 \mathrm{~km}$ at the magnetopause.

Separatrix regions are important for the coupling between the Earth's magnetosphere and the ionosphere, as they connect X-lines at the magnetopause to the ionosphere. It has been shown that separatrix regions can extend far from the X-line and still keep their narrow (ion inertial length scale) width as well as strong currents and electric fields (Khotyaintsev et al., 2006). Possible manifestations of the separatrix regions are strong auroras at the plasma sheet boundary layer (PSBL) for reconnection in the magnetotail and poleward moving auroral forms, PMAFs, (Sandholt et al., 1998, 2002) for reconnection at the dayside magnetopause.

Earlier studies of separatrix regions have led to a significant improvement in understanding of the basic structure of these regions. It has been shown that ions are not "frozen-in", $\boldsymbol{E}+\boldsymbol{v}_{i} \times \boldsymbol{B} \neq 0$, in the separatrix region (Khotyaintsev et al., 2006). The deviation from the "frozen-in" condition occurs on scales comparable to or smaller than the ion inertial length, $\lambda_{i}=c / \omega_{p i}$, and is mainly provided by the Hall term and electron pressure effects in the generalized Ohm's law (André et al., 2004; Vaivads et al., 2004b; Khotyaintsev et al., 2006). The separatrix regions are regions of strong electric fields, currents and wave activity. As in the case of the slow shock, plasma heating and energization takes place in the separatrix region (Wygant et al., 2005; Vaivads et al., 2006; Topliss et al., 2001; Bogdanova et al., 2004).

One characteristic feature of separatrix regions is the existence of a density cavity inside the region (Mozer et al., 2002; André et al., 2004; Wygant et al., 2005; Cattell et al., 2005; Retinò et al., 2006; Khotyaintsev et al., 2006). Numerical simulations also predict a density cavity as well as 
strong currents and electric fields within the separatrix region (Shay et al., 2001; Pritchett and Coroniti, 2004). Several mechanisms have been suggested to explain the formation of the cavity: electron density decrease close to a separatrix can be caused by escape of energetic (hot) magnetospheric electrons to the magnetosheath along a newly opened field line (Khotyaintsev et al., 2006) and/or due to parallel electric fields at the separatrix (Cattell et al., 2005). At the same time, the decrease of ion density within the cavity can be due to the acceleration of ions by the strong electric fields in the normal direction as ions move from the magnetosphere into the outflow region (Shay et al., 2001; Khotyaintsev et al., 2006).

Systematic statistical studies addressing the internal structure and the electric and magnetic fields of the separatrix regions have so far been lacking. Here we present a study of multiple encounters of separatrix regions by the Cluster spacecraft during ongoing reconnection at the magnetopause. We investigate if the features of the separatrix region identified in single event studies (the density cavity, strong current and potential jump) are typical for most of the separatrix region crossings.

\section{Data set}

We analyze data from the four Cluster spacecraft during the time interval 12:30-14:30 UT on 4 January 2004. The spacecraft position is shown in Fig. 2 together with geomagnetic field lines obtained from the Tsyganenko T96 model (Tsyganenko, 1995). Cluster is located on the dusk side in the Northern Hemisphere sunward of the cusp. The separations between the spacecraft are small, about $300 \mathrm{~km}$.

During the time interval we have studied, Cluster is in burst mode with high sampling rates on all instruments. We use data from all spacecraft. The electric field is obtained from the EFW instrument (Gustafsson et al., 2001). EFW measures two components of the electric field in the spacecraft spin plane low-pass filtered at $180 \mathrm{~Hz}$ and sampled at $450 \mathrm{~Hz}$. We use plasma density derived from the spacecraft potential, available at a resolution of $5 \mathrm{~Hz}$, using an empirical conversion law (Escoubet et al., 1997; Pedersen et al., 2008) and calibrated using electron density measured by PEACE. Thus we can obtain plasma density estimates at a higher temporal resolution than from particle instruments where the data must be integrated over the spin period of $4 \mathrm{~s}$ to obtain plasma density. The magnetic field data is from the fluxgate magnetometer (FGM) (Balogh et al., 2001). FGM has a sampling frequency of $67 \mathrm{~Hz}$. The electron data is from the plasma electron and current experiment (PEACE) (Johnstone et al., 1997; Owen et al., 2001) on spacecraft C2. We have used the 3DXP product from the high energy electron analyzer (HEEA), which covers the energy range from $30 \mathrm{eV}$ to $26 \mathrm{keV}$. The ion data is from the hot ion analyser part of the Cluster ion spectrometry experiment (CIS-HIA) (Rème et al., 2001) on $\mathrm{C} 1$, which measures the ion fluxes in the energy
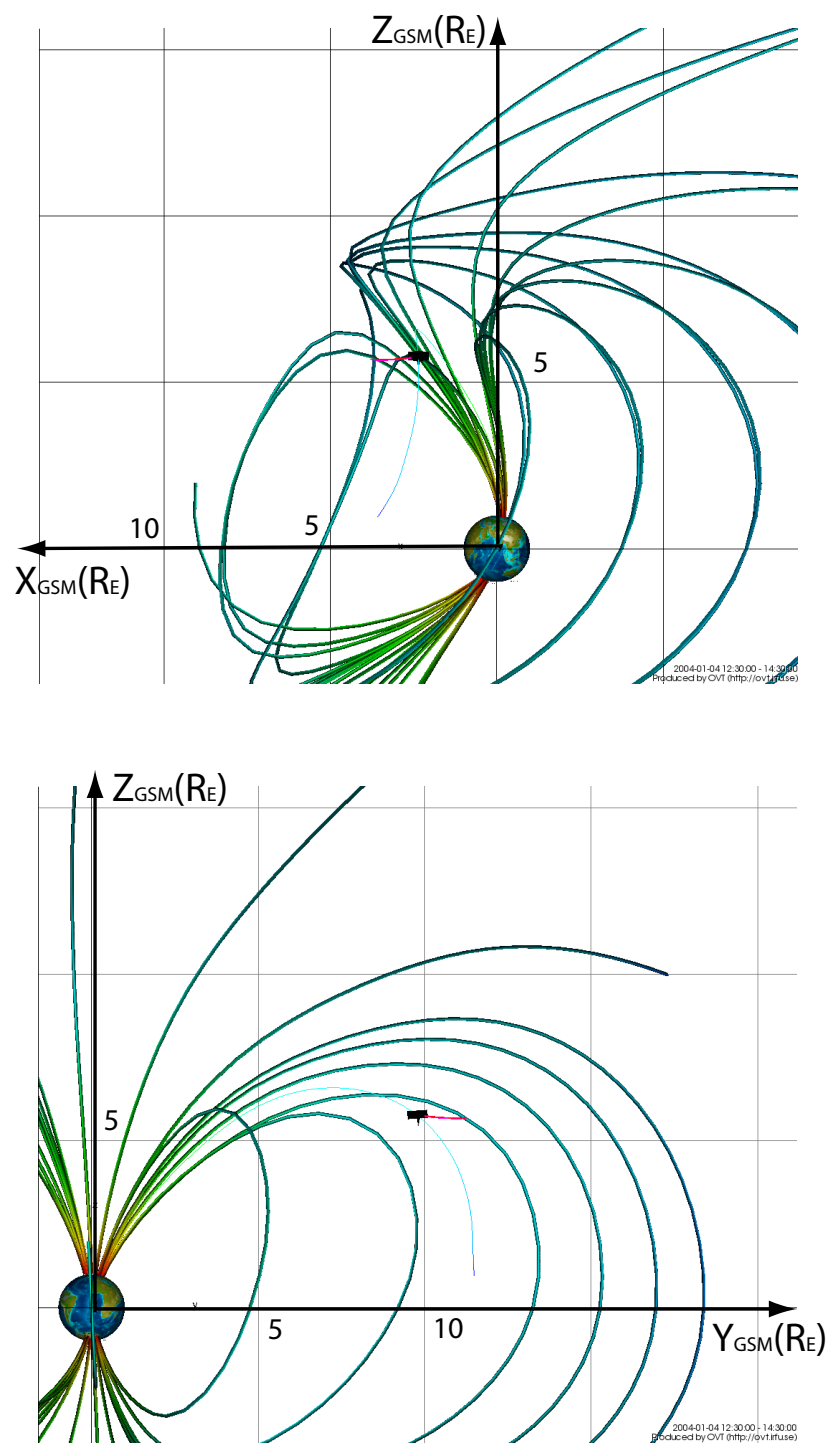

Fig. 2. Cluster orbit on 4 January 2004, 12:30-14:30 UT. During this time interval Cluster moved from [2.3 9.8 5.7] $R_{E}$ (GSM) to [ $\left.\begin{array}{lll}3.7 & 11.2 & 5.7\end{array}\right] R_{E}$ (GSM). The maximum separation between the spacecraft is $\sim 300 \mathrm{~km}$. The plot was created using OVT (http://ovt. irfu.se).

range from 5 to $32000 \mathrm{eV} / \mathrm{e}$. From the CIS-HIA instrument we also use the spin averaged ion velocity moment.

An overview of ACE (Smith et al., 1998; McComas et al., 1998) and Cluster data during the interval 12:30-14:30 UT on 4 January 2004 is presented in Fig. 3. The interplanetary magnetic field (IMF) z-component, measured by the MAG instrument on the ACE spacecraft, is shown in Fig. 3a together with the IMF clock angle $\theta=\arctan \left(B_{y} / B_{z}\right)$. This means that $0^{\circ}$ and $360^{\circ}$ correspond to positive $B_{z}, 90^{\circ}$ to positive $B_{y}, 180^{\circ}$ to negative $B_{z}$ and $270^{\circ}$ to negative $B_{y}$. The IMF is predominantly southward, with $B_{z}$ (GSM) varying 


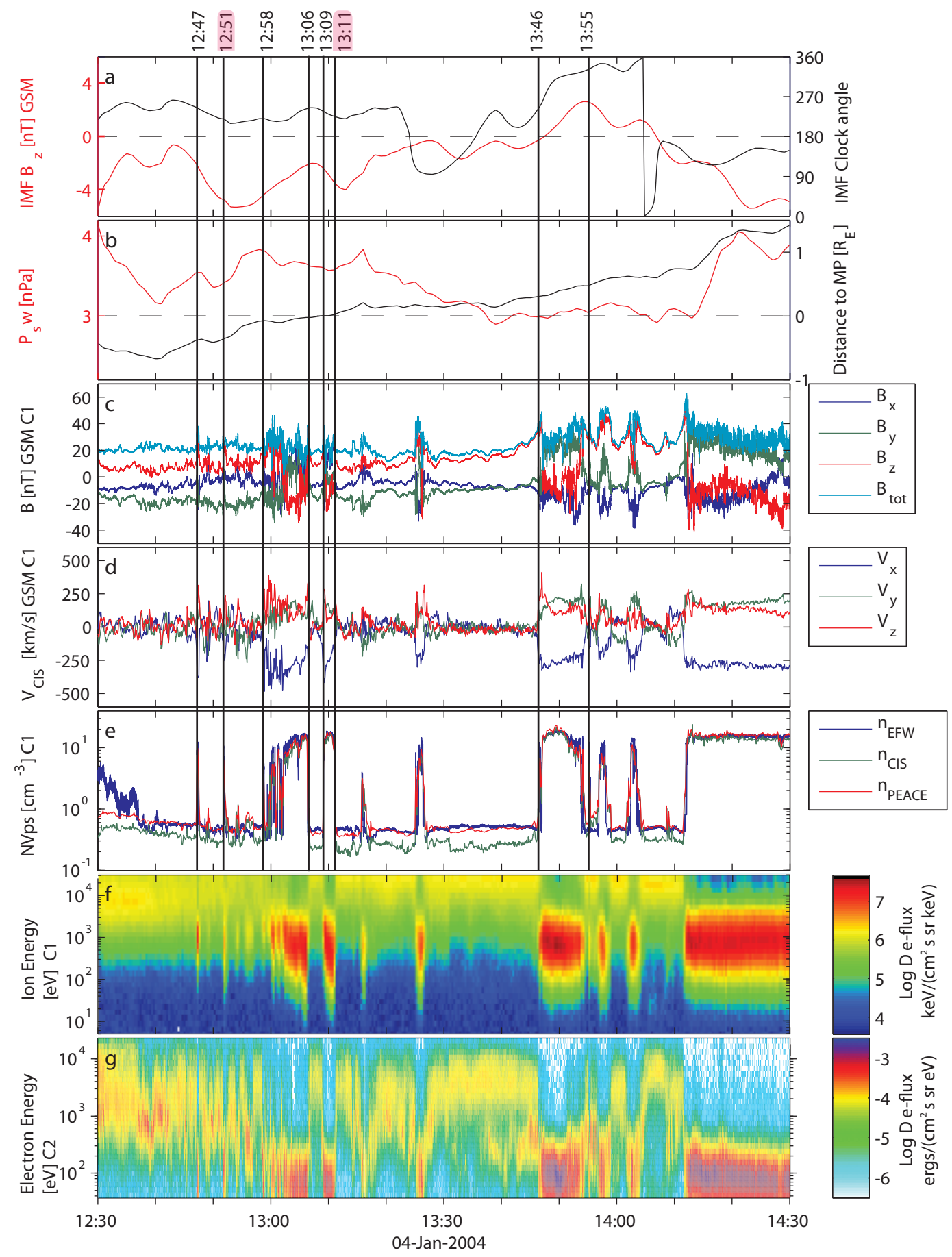

Fig. 3. Overview of the Cluster magnetopause crossing on 4 January 2004, 12:30-14:30 UT from SC1. Panel (a) shows the interplanetary magnetic field (IMF) $B_{z}$ and the clock angle observed by the ACE spacecraft. Panel (b) shows the solar wind pressure and the estimated distance to the magnetopause. Panels (c-g) contain data from Cluster. Panel (c) shows the GSM components and magnitude of the magnetic field. Panel (d) shows the ion velocity in GSM coordinates. Panel (e) shows three different plasma density estimates based on the spacecraft potential, electron instrument PEACE and ion instrument CIS data. Panel (f) shows the ion differential energy flux spectrogram. Panel (g) shows the electron differential energy flux spectrogram. The vertical lines mark sub-intervals which are analyzed in detail and summarized in Table 1. Details for the sub-intervals marked in pink are shown in Figs. 8 and 9. 
between $-8 \mathrm{nT}$ and $4 \mathrm{nT}$. In Fig. $3 \mathrm{~b}$ we show the solar wind dynamic pressure together with the distance between the Cluster location and the model magnetopause calculated using the Shue model (Shue et al., 1997). The negative distance corresponds to locations inside the magnetosphere, as it is at the beginning of the interval, and the positive distance corresponds to the location inside the magnetosheath. ACE is located at the Lagrangian point L1 between the Sun and Earth. The time delay of the solar wind data is estimated with the method described by Weimer et al. (2003) with modifications by Haaland et al. (2006).

Figure $3 \mathrm{c}-\mathrm{g}$ shows Cluster data during the same interval 12:30-14:30 UT. In the first half of the interval, between 12:30 and 13:45 UT, Cluster is located primarily inside the magnetosphere, and after a number of magnetopause crossings (change of sign of $B_{y}$ and $B_{z}$ between 12:45 and 14:15 UT, Fig. 3c) exits to the magnetosheath at 14:12 UT. At the magnetospheric side the magnetic field is directed primarily along the $+B_{z}$ and $-B_{y}$ GSM. The magnetospheric plasma is stagnant (flow velocity is close to zero) and has a relatively low density $\sim 0.5 \mathrm{~cm}^{-3}$ (Fig. 3d). The ion spectrogram at spin resolution in Fig. 3f show omni-directional ion energy fluxes. Magnetospheric ions (Fig. 3f) and electrons (Fig. 3g) are hot, their temperatures are a few $\mathrm{keV}$ and $\sim 10 \mathrm{keV}$ respectively. In the magnetosheath the conditions are very different. The magnetic field is directed primarily along the $-B_{z}$ and $+B_{y}$ GSM. The plasma is relatively dense, $\sim 14 \mathrm{~cm}^{-3}$, and flowing around the magnetopause (negative $V_{x}$, and positive $V_{y}$ and $V_{z}$ in Fig. 3d) at $\sim 350 \mathrm{~km} / \mathrm{s}$. The temperature of ions is $\sim 400 \mathrm{eV}$ and electrons $\sim 100 \mathrm{eV}$.

\section{Reconnection evidence}

In this section, we discuss large scale evidence of ongoing reconnection during the selected time interval when Cluster is located close to the magnetopause. Then we illustrate the motion of the flux tubes assuming reconnection is occurring. The kinetic evidence of reconnection based on the electron distribution properties is discussed in the next section where two crossings of separatrix region are presented in detail.

Earlier studies show that reconnection at the magnetopause can be associated with flux transfer events (FTEs), bulges due to varying magnetic reconnection rate propagating along the magnetopause (Russell and Elphic, 1978; Scholer, 1995; Khotyaintsev et al., 2004). The multiple crossings of the magnetopause we observe can also be caused by FTEs. Comparing magnetopause crossings with solar wind pressure variations in Fig. 3b we find no clear correlation and thus rule out solar wind pressure variations as a cause of most magnetopause crossings. During the first part of the interval Cluster is primarily located inside the magnetosphere, the FTEs can be identified in Fig. 3 as encounters of magnetosheath-like plasma on the magnetospheric

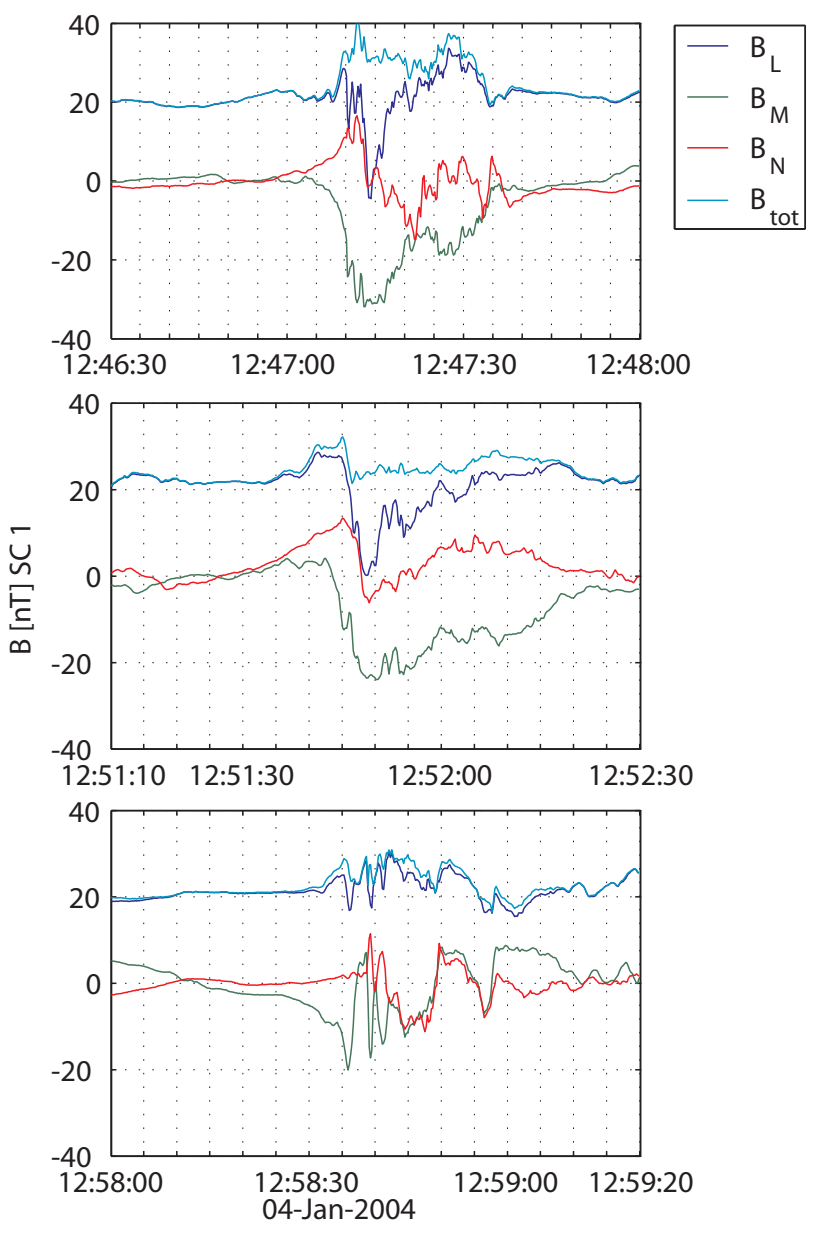

Fig. 4. The magnetic field in local $L M N$-coordinates from Cluster 1 for the events $12: 47,12: 51$ and 12:58, where $B_{L}$ is dark blue, $B_{M}$ is green, $B_{N}$ is red and $B_{T O T}$ is light blue. The $N$-direction here is the normal to the magnetopause for the entry into the magnetosheath at 14:10. This is direction is very similar to the local timing on the parallel current sheet used in the analysis. The $L$-direction is the local magnetospheric direction.

field lines, e.g. at 12:47, 12:51, 12:58 UT. At these times the magnetic field increases in magnitude, however does not change to the magnetosheath direction. The ion velocity is sometimes as high as $\sim 500 \mathrm{~km} / \mathrm{s}$ and the plasma density increases almost to the magnetosheath level. The plasma is a mixture of magnetospheric and magnetosheath populations. The magnetic field in local $L M N$-coordinates from Cluster 1 for the events 12:47, 12:51 and 12:58 are shown in Fig. 4. The normal component of the magnetic field $B_{N}$ (red, normal direction is determined by inter-spacecraft timing on the full magnetopause crossing at 14:10) shows a bipolar signature characteristic for FTEs (Russell and Elphic, 1978; Owen et al., 2008). $B_{N}$ changes from positive to negative, which is consistent with northward and tailward propagation of FTEs. The event at 12:51 UT shows a typical bipolar signature of 


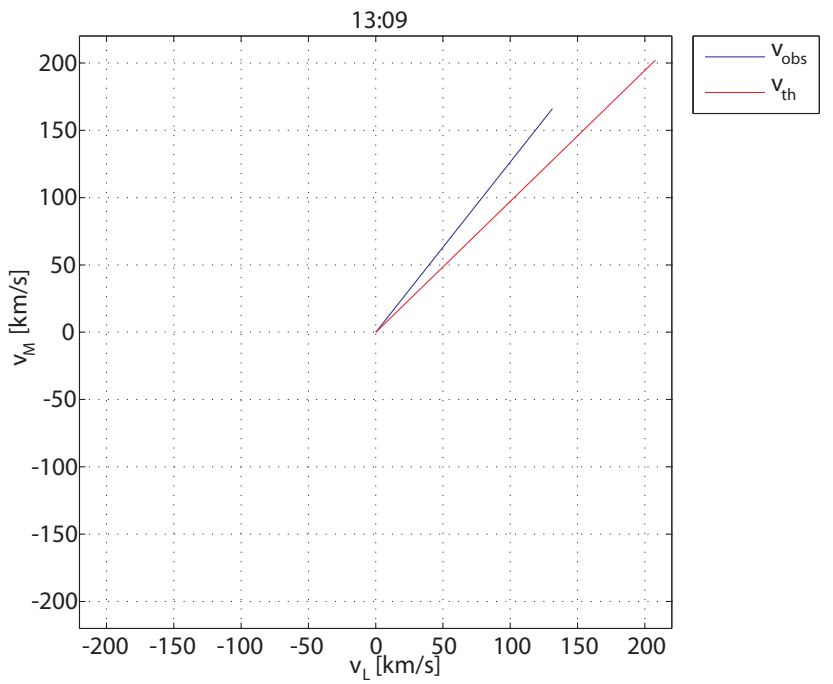

Fig. 5. Results of the Walén test for the event at 13:09 UT. The outflow region is observed for more than $10 \mathrm{~s}$ and the result indicate that reconnection is ongoing sunward of the spacecraft. The blue line corresponds to the observed velocity and the red line to the calculated velocity.

an FTE traveling tailward, and the event at 12:47 UT is very similar. The event at 12:58 UT has a more complicated structure of the normal magnetic field component; this structure, however, is likely to be produced by the same process as the FTE and can be interpreted as overlaid bipolar signatures.

To test for the presence of reconnection locally we have also carried out the Walén test (Sonnerup et al., 1981) for the events where there is data from both the outflow region and the magnetosheath, i.e. when the spacecraft cross the magnetopause. Figure 5 shows the Walén test for the magnetopause crossing at 13:09 UT. Figure 5 shows a comparison of the measured and estimated change of the velocity $v_{L}$ and $v_{M}$ components. The ratio between the absolute values of the predicted and the observed magnitudes of the velocity vectors is 0.73 and the angle between the vectors is $7^{\circ}$. The event at 13:09 UT shows a good agreement between the observed and predicted ion velocities and we conclude that within the magnetopause there is likely a rotational discontinuity with the reconnection X-line located sunward of the spacecraft. For the events 13:06 and 13:11 UT the crossings of the outflow region are very fast and ion moments are measured only in at most 1-2 points inside the outflow region. However, these few points cannot be fully trusted because the ion moments are computed from the data collected during one spin $(\sim 4 \mathrm{~s})$, and both the magnetic field and plasma density are highly variable at such time scales. Thus, we do not expect a good Walén test for these events. In summary, a reliable Walén test was only possible to perform on one crossing and this test suggest that the spacecraft are passing reconnecting flux tubes that has been reconnecting sunward from the spacecraft in all of the three analyzed crossings.
Both the polarity of the observed FTEs and the Walén test indicate that the reconnection site is located sunward from the spacecraft and this location is further supported by the Cooling model (Cooling et al., 2001). The Cooling model predicts the motion of reconnected flux tubes by calculating their velocity. This is the velocity of the de Hoffman-Teller frame calculated from the magnetosheath velocity, magnetic field and density. The magnetosheath magnetic field is from a model by Kobel and Fluckiger (1994) where characteristic solar wind magnetic field values for the time interval 12:50-13:10 UT is used. The geomagnetic field used by Cooling et al. (2001) to estimate the magnetopause current is very simple and the location of the anti-parallel $\mathrm{X}$-line is approximate. We have made one run of the Cooling model for characteristic solar wind conditions during the interval (southward-duskward pointing IMF). In Fig. 6 we show the predictions of flux tube motion for the location of the reconnection X-line at the subsolar point (in Fig. 6a) and where the reconnecting fields are antiparallel (in Fig. 6b). In the first case, where we assume that the reconnection occurs near the subsolar point, see Fig. 6a, the flux tubes connected to the Northern Hemisphere move poleward and duskward (red paths) and pass the location of the spacecraft. In the second case, see Fig. 6b, we assume that reconnection sites are in the pre-noon sector of the Northern Hemisphere and post-noon sector of the Southern Hemisphere, as expected from antiparallel reconnection (Crooker, 1979), and the model predicts that we should not expect reconnected flux tubes to pass the location of the spacecraft. Thus, we conclude that for characteristic IMF conditions during the interval the reconnection $\mathrm{X}$-line is most probably located close to the subsolar point.

The FTE signatures and the Walén test show the presence of ongoing reconnection with an X-line located southward of the spacecraft during the major part of the studied interval with southward IMF. The Cooling model results indicate that for these IMF conditions the reconnection X-line is located near the sub-solar region. Note that there is additional evidence for Cluster crossing reconnected flux tubes related to the properties of the separatrix regions themselves and that is discussed in the next section, e.g. the mixture of magnetosheath and magnetospheric electrons.

\section{Observations of separatrix regions}

We have made a detailed analysis of the separatrix region on the magnetospheric side of the magnetopause reconnection layer (see Fig. 1). Eight crossings or partial entries into the magnetopause reconnection layer were selected and are marked by vertical lines in Fig. 3. The crossings were selected such that all four spacecraft observe similar signatures in magnetic field and density, thus making it possible to perform multi-spacecraft timing analysis (Schwartz, 2000). The events at 12:47, 12:51, 12:58, 13:46 and 13:55 UT are partial 

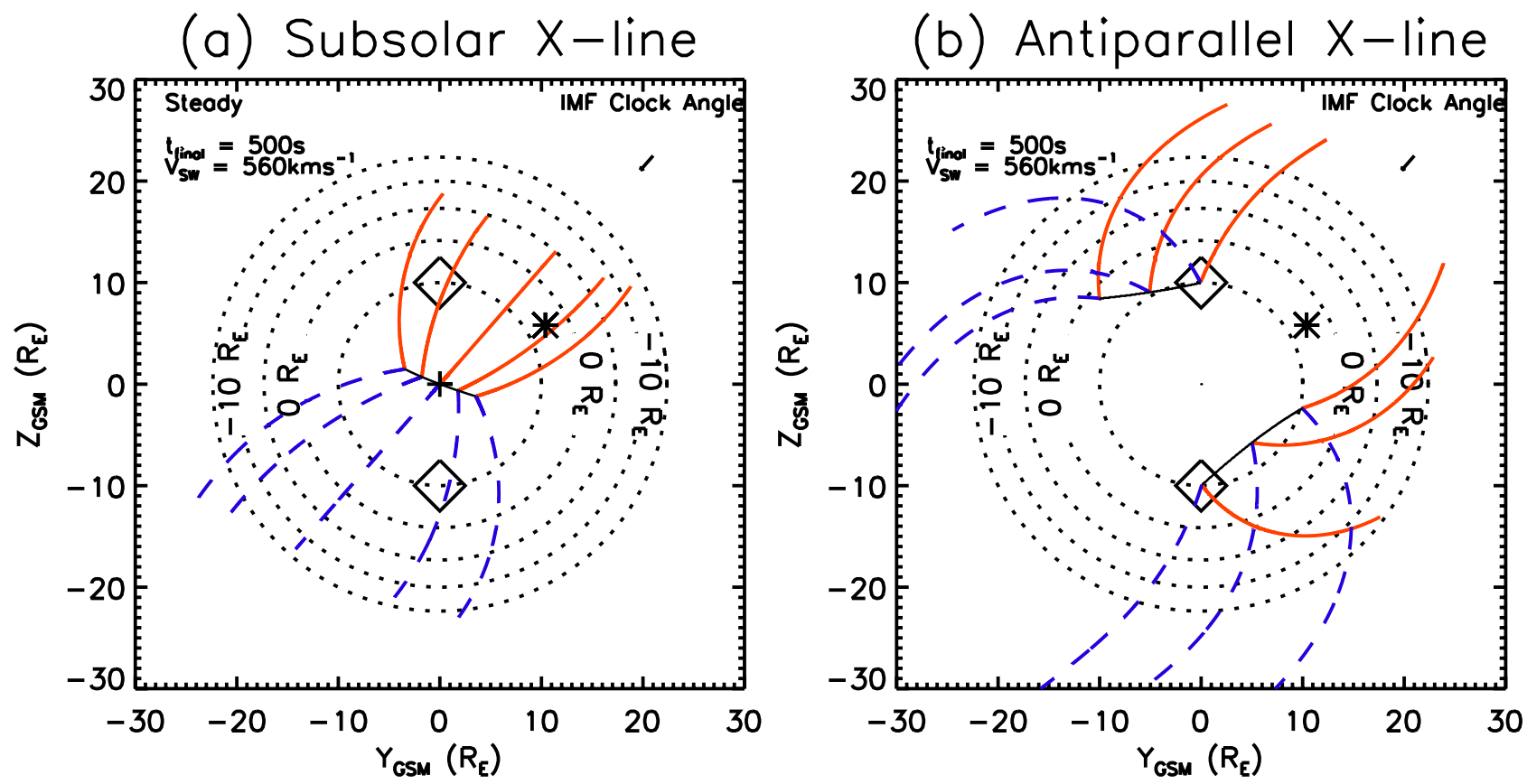

Fig. 6. Motion of the opened field lines predicted by the Cooling model. Field lines connected to the Northern Hemisphere are shown in red and field lines connected to the Southern Hemisphere are shown in blue. In (a) reconnection is initiated near the subsolar point. In (b) reconnection is initiated in region of highest shear in the Northern and Southern Hemisphere. The position of Cluster is shown with an asterisk. The diamond show the location of the cusp. The dotted lines are contours in $X_{\mathrm{GSM}}$.

entries into regions with magnetosheath-like plasma located on magnetospheric field lines; the dominant magnetic field component $B_{z}$ does not change sign throughout the crossing and thus the magnetic field preserves its magnetospheric orientation. The first event, at 12:47 UT, was analyzed in detail by Khotyaintsev et al. (2006). Note that some of these events may look like full crossings of the magnetopause reconnection layer in the overview plot (Fig. 3), e.g. 13:46 UT, but this is due to a short time delay between a partial and a full crossing. In the other events, at 13:06, 13:09 and 13:11 UT in Fig. 1, the spacecraft fully cross the main magnetopause current layer (identified by the change of $B_{z}$ sign). We find similar characteristics for the eight events we have studied and present detailed data for the two sub-intervals (at 12:51 and 13:11 UT) marked with pink on top of Fig. 3. These two cases represent the two different situations: a partial (12:51 UT) and a full (13:11 UT) crossing of the magnetopause reconnection layer.

\subsection{Full crossing of the magnetopause reconnection layer, 13:11 UT}

The observations are presented in the local $L M N$-coordinate system of the magnetopause obtained from minimum variance analysis (MVA). The $N$ direction given by MVA is consistent with normal direction obtained from timing analysis of the magnetopause crossing (both density and mag- netic field). Observations of the magnetopause crossing at 13:11 UT are shown in Fig. 7. The panels from top to bottom show: plasma density from EFW spacecraft potential (Fig. 7a), $L M N$-components of the magnetic field from FGM (Fig. 7b), $N$ (Fig. 7c) and $L$ (Fig. 7d) components of plasma flow velocity, full (green) and perpendicular (red) from CISHIA and $\boldsymbol{E} \times \boldsymbol{B}$ from EFW, antiparallel (Fig. 7e) and parallel (Fig. 7f) to $B$ electron fluxes from the PEACE 3DXP product (HEEA sensor) which has been rebinned using the actual magnetic field measured by the FGM.

Cluster is located in the magnetosheath (high density side) in the beginning of the interval and plasma flow is nearly parallel to $B$. Then plasma convection in the $N$ direction increases (magnetopause moves outward) and Cluster crosses the main magnetopause current layer between 13:10:58 and 13:11:06 UT where $B_{L}$ changes sign. On scales larger than ion inertial length $v_{\mathrm{EFW}} \sim v_{\mathrm{CIS} \perp}$ and this velocity reflects the overall motion of the magnetopause (marked by the green bar in Fig. 7) in the normal direction. Inside this current layer there is also an accelerated plasma flow in the $L$ direction and we can identify the outflow/field reversal region (green bar marked OR in Fig. 7) bounded by the ion edges (purple bars). The ion edge on the magnetospheric side is identified at the main density gradient, that is the edge of the bulk flow of the reconnection jet. On the magnetosheath side the ion edge is located outside the field reversal region 


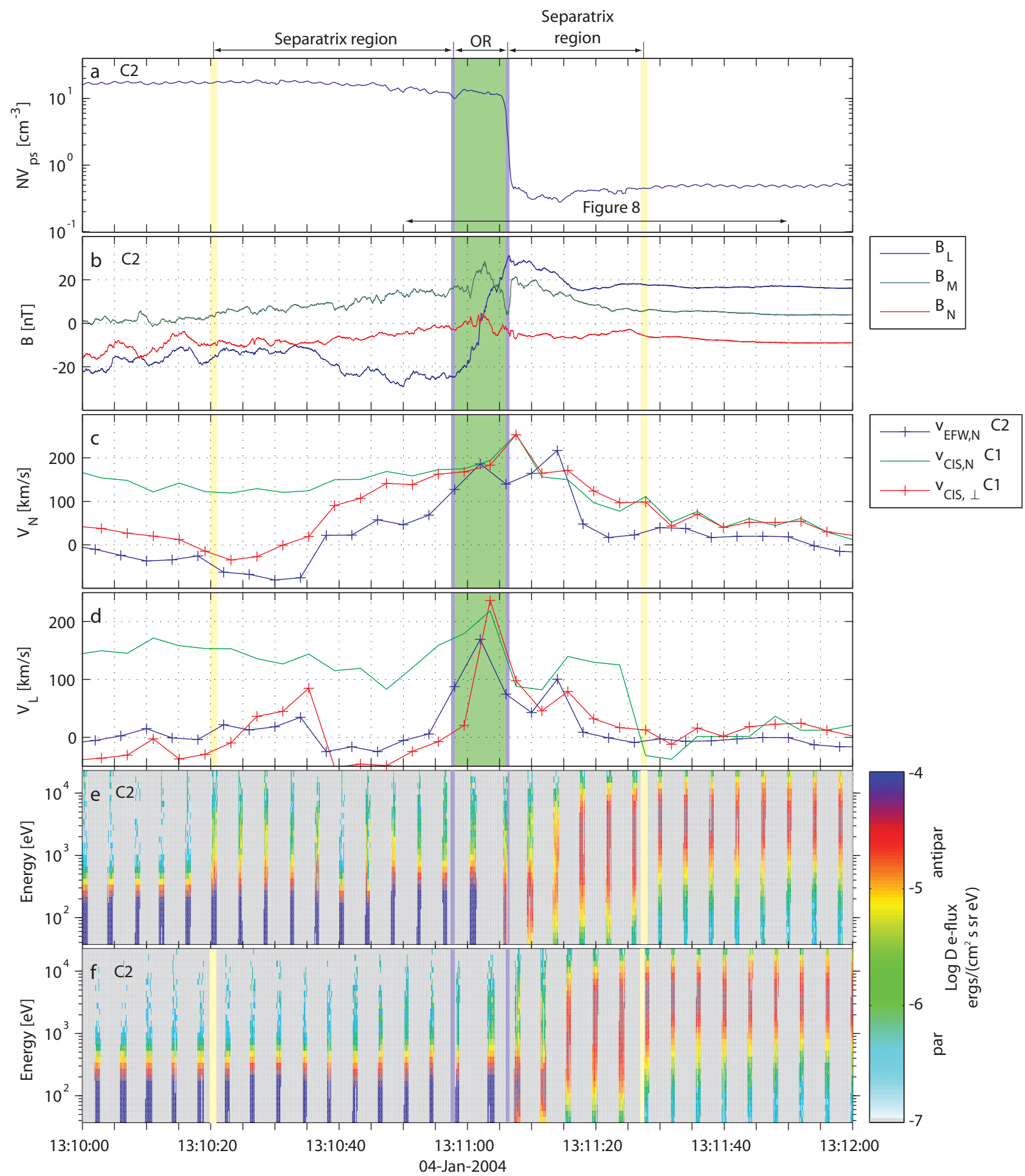

Fig. 7. Cluster crossing of the magnetopause reconnection layer on 4 January 2004, 13:11 UT. The panels from top to bottom show: (a) plasma density, (b) $L M N$-components of the magnetic field, (c) $v_{N}$ normal plasma flow velocity, (d) $v_{L}$ flow velocity components of plasma flow velocity (full and perpendicular), (e) electron flux anti-parallel to $B$ and (f) electron flux parallel to $B$. The $L M N$-directions

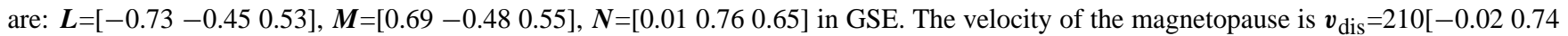
$0.67] \mathrm{km} / \mathrm{s}$ GSE. Vertical green bar marks the outflow/field reversal region (OR), and yellow bars mark the outer and inner separatrices. Arrow in the top panel marks the time interval presented in detail in Fig. 8. 
at the edge of the ion jet. $B_{N}$ is negative, which is consistent with reconnection $\mathrm{X}$-line being sunward from the spacecraft. While in the magnetosheath, at 13:10:20 UT Cluster detects a sharp increase in anti-parallel (away from the magnetopause) electron flux at energies above $400 \mathrm{eV}$, i.e. electrons of magnetospheric origin. At the same time there is no change in the parallel flux. We identify this point as the outer separatrix or magnetosheath electron edge (E1), see Fig. 1. In the separatrix region on the magnetospheric side of the magnetopause, 13:11:06-13:11:28 UT, we observe a mixture of magnetosheath-like electrons and electrons with energies 1$10 \mathrm{keV}$, similar to the magnetospheric electron population. The transition to purely magnetospheric populations happens at 13:11:28 UT. We identify this point as the inner separatrix or magnetospheric electron edge (E2).

We further investigate the details of the reconnection layer, particularly concentrating on its magnetospheric side. Figure 8 shows detailed observations. Figure $8 \mathrm{a}-\mathrm{d}$ shows $L M N$ components and magnitude of the magnetic field. Figure $8 \mathrm{e}$ shows density derived from the EFW spacecraft potential. Figure 8f shows the ion energy spectrogram. Note that this ion spectrogram does not display the spin-averaged flux; it shows snapshots of the ion energy flux spectra for sub-spin accumulation periods of $\sim 0.25 \mathrm{~s}$. The fact that ion energy fluxes vary in a periodic fashion simply reflects the presence of a bulk ion flow relative to the spacecraft which appears modulated due to the spacecraft spin. Figure 8g shows current parallel to the magnetic field. The observed localized current sheets are too narrow to use multi-spacecraft techniques such as the curlometer (Robert et al., 2000) to estimate their current density. Instead, we estimate the current using a single spacecraft method (Luhr et al., 1996): we assume that changes in the magnetic field are due to plane current sheets moving across the spacecraft with a velocity, $\boldsymbol{v}_{\text {dis }}$, which is determined from timing analysis. Figure 8 h shows the $N$ component of the electric field from EFW. Figure 8i shows the electric potential which is obtained by integrating the $\mathrm{N}$ component of the electric field across the boundary. The full E-field vector is computed from the 2-D electric field measured by EFW using the zero parallel electric field assumption. Figure $8 \mathrm{j}-\mathrm{k}$ show the electron differential energy flux in two directions. The flux is obtained from the PEACE 3DXP product (HEEA sensor) which has been rebinned using the actual magnetic field measured by the FGM. Then we select the sectors being perpendicular (Fig. 8j) and with the smallest measured angle relative to the magnetic field (the most parallel direction, Fig. 8k). The observed angle between the selected sector with the smallest pitch angle and the magnetic field is shown in Fig. 81 (blue line). The sector with the smallest pitch angle is often rather far from being parallel to the magnetic field (up to $60^{\circ}$ ) and the direction parallel to $B$ is generally sampled only once per spin (angle $\sim 0^{\circ}$ ). Since the electron distribution is anisotropic, the changing pitch angle of the parallel sector recorded in Fig. 8k produces a characteristic periodic pattern. Figure $8 \mathrm{~m}$ shows distance to the magnetopause obtained by integration of the $N$-component of the plasma convection velocity, $(\boldsymbol{E} \times \boldsymbol{B})_{N}$, shown in Fig. 7c. The time axes of spacecraft SC1, SC3 and SC4 have been time shifted so that the different spacecraft data can be compared in the boundary reference frame.

The magnetopause current layer is identified by a change of $B_{L}$ from negative in the magnetosheath to positive in the magnetosphere. Inside this current layer $B_{M}$ makes a bipolar change (Fig. 8b). Such bipolar signatures are usually attributed to Hall magnetic fields (Vaivads et al., 2004b). The magnetospheric edge of the $B_{M}$ variation coincides with the main magnetopause density gradient, at which the density drops from the magnetosheath to magnetospheric level, and this is also the location of the ion edge (marked by the purple bar). At the density gradient there is a strong current parallel to the magnetic field (Fig. 8g); the current density is $0.2 \mu \mathrm{A} / \mathrm{m}^{2}$. The parallel current is produced by Hall currents flowing towards and away from the $\mathrm{X}$-line, in this case the strongest parallel current is flowing towards the $\mathrm{X}$-line.

The high energy magnetospheric ion population is present on both sides of the magnetopause current layer (Fig. 8f). The magnetospheric population starts at several $\mathrm{keV}$ and goes outside the CIS-HIA energy range. The magnetospheric edge of the ion jet is located at 13:11:06 UT, at the main density gradient. At the high density side the plasma is dominated by the magnetosheath population with a typical energy of several hundred $\mathrm{eV}$. As the spacecraft cross the density gradient it moves into a region populated mainly by plasma of dayside plasma sheet origin. At the same time (13:11:06 UT) the $L$-component of the plasma flow velocity decreases (Fig. $7 \mathrm{~d})$. The ion jet $\left(V_{L} \sim 200 \mathrm{~km} / \mathrm{s}\right)$, accelerated by the $\boldsymbol{J} \times \boldsymbol{B}$ force, is localized inside the magnetopause current layer and is directed mostly perpendicular to $B$. On the magnetospheric side the main density gradient bounds the ion jet and we identify it as the "ion edge" (I2), see Fig. 1. Some magnetosheath ions with flux lower than in the magnetosheath are still seen within the gyroradius distance from the density gradient, $\rho_{i} \sim 230 \mathrm{~km}$, and even deeper inside the magnetosphere (seen around 13:11:22 UT in Fig. 8f, and a corresponding secondary velocity peak in $V_{L}$ in Fig. $7 \mathrm{~d}$ ). The ion edges bound the outflow region observed between 13:10:58-13:11:06 UT where the plasma flow $L$-component increases in Fig. 7. On the magnetosheath side the ion edge cannot be well defined due to the data resolution and the larger gyroradius of magnetospheric ions. A good estimation of the position of the ion edge on the magnetosheath side is at the start of the accelerated ion flow, i.e. where the longitudinal plasma flow, $V_{L}$, in Fig. 7 increases as the spacecraft enter the outflow region from the magnetosheath.

Prior to the magnetopause current layer crossing we observe mainly the magnetosheath electron population in Fig. 8j and Fig. 8k, with electron energies up to $200 \mathrm{eV}$ $\left(T_{e} \sim 40 \mathrm{eV}\right)$. On the magnetospheric side of the magnetopause current layer (on the low density side) the magnetosheath population is of much lower density, and it is mixed 


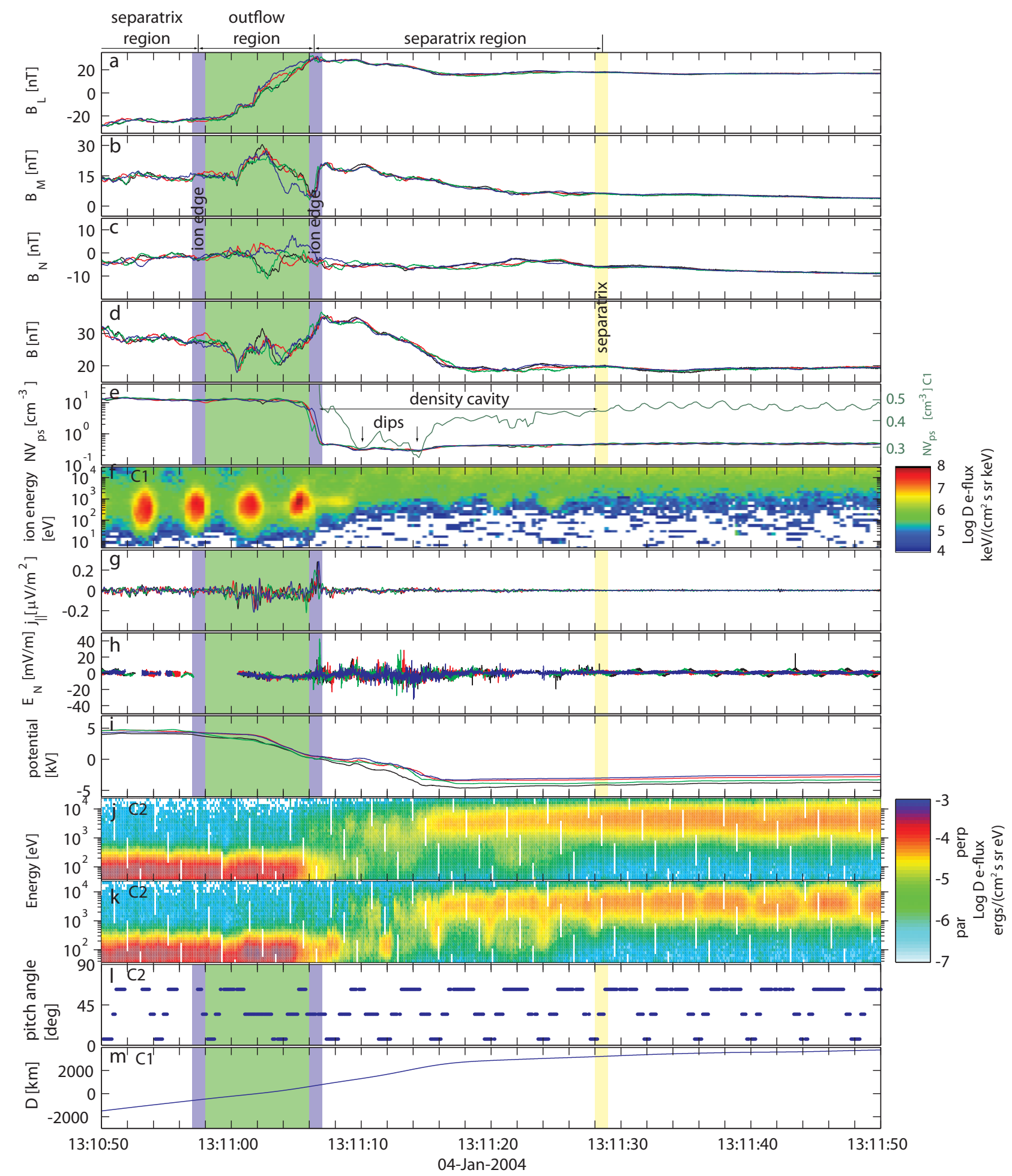

Fig. 8. Cluster crossing of the magnetopause reconnection layer on 4 January 2004, 13:11 UT. Panels (a-d) show the magnetic field $L M N$ components and magnitude of the magnetic field for all four spacecraft. Panel (e) shows the density and the density on a different scale from $\mathrm{C} 1$, (f) the energy spectrogram of ions over all angles from $\mathrm{C} 1,(\mathbf{g})$ the current density parallel to the magnetic field, (h) the normal electric field, (i) the electric potential, (j) the energy spectrogram of electrons perpendicular to the magnetic field from $\mathrm{C} 2$, (k) the energy spectrogram of electrons with the smallest measured pitch angle, from $\mathrm{C} 2$ and (l) the pitch angle of electrons in panel (k), (m) the distance to the magnetopause. The black, red, green and blue lines represent spacecraft 1, 2, 3 and 4, respectively. The $L M N$-directions are the same as in Fig. 7. The time axes for spacecraft $1-4$ are shifted by $\left[\begin{array}{llll}1.0 & 0 & 0.5 & -0.3\end{array}\right] \mathrm{s}$. 
with the magnetospheric population (energies above $2 \mathrm{keV}$, $T_{e} \sim 2 \mathrm{keV}$ ). Alongside with high energy (above $2 \mathrm{keV}$ ) magnetospheric electrons, electrons in the intermediate energy range $0.2-2 \mathrm{keV}$ (above typical magnetosheath energy and below the magnetospheric) are observed between 13:11:06 (magnetospheric ion edge) and 13:11:28 UT, which we have identified as "magnetospheric separatrix". The region between the separatrix and the ion jet, is the "separatrix region". At the magnetospheric side of the separatrix (after 13:11:28 UT) electrons have a typical distribution with a narrow loss cone, calculated to be $\sim 2^{\circ}$. The loss cone appears as periodic pattern in the parallel electrons (Fig. 8k) observed after 13:11:28 UT; the pattern is created due to a variation of the detector angle with respect to $\boldsymbol{B}$ : minima are seen when the angle (Fig. 81) is close to zero. When plasma crosses the separatrix from the closed magnetic field lines of the magnetosphere to the open field lines of the separatrix region, parallel magnetospheric electrons start to escape to the magnetosheath creating a wider loss cone. At the same time also the magnetosheath electrons enter the separatrix region along the open field lines. During the magnetopause current layer crossing these electrons can be further accelerated into the intermediate energy range $0.2-2 \mathrm{keV}$ that is higher than typical electron energies in the magnetosheath. Such electrons with parallel energies less than $2 \mathrm{keV}$ are observed at 13.11:28, 24, 20 UT, e.g. at times when PEACE samples the most field aligned population (pitch angle $\sim 0^{\circ}$ in Fig. 81). We locate the separatrix around 13:11:28 UT where the most energetic parallel electrons start to disappear and the accelerated magnetosheath electrons appear.

The separatrix region is $2400 \mathrm{~km}$ wide (Fig. $8 \mathrm{~m}$ ) and it coincides with a region of increased magnetic field magnitude and decreased plasma density (cavity, Fig. 8e). However, it is important to notice that the density is derived from the spacecraft potential and changes in the spacecraft potential are mainly related to variations in electron density, but also depend slightly on variations in electron temperature. Therefore on boundaries separating plasmas of different temperatures and densities, as in our case the separatrix region, the estimation of the density from the spacecraft potential is more complicated and the absolute depth of the density cavities needs to be further investigated. The density/spacecraft potential cavities are characteristic for separatrix regions on the magnetospheric side and they can even be useful in the identification of separatrix regions. In the cavity there are at least two individual density dips (Fig. 8e), each a few seconds long. Multi-spacecraft data allow us to see that the observations of the individual density dips are time lagged among the spacecraft in such a way that we can conclude that the spacecraft cross multiple density dips and do not make multiple encounters of the same density dip.

Inside the separatrix region there are strong normal electric fields ( $E_{N}$, Fig. 8 h) up to $30 \mathrm{mV} / \mathrm{m}$. The estimated change of the electric potential across separatrix region is $4 \mathrm{kV}$ between 13:11:05-13:11:16 UT. The small distance between
13:11:16-13:11:28 UT does not affect the electric potential considerably.

\subsection{Partial crossing of the magnetopause reconnection layer, 12:51 UT}

The event at 12:51 UT (partial BL crossing, FTE) is presented in Fig. 9 (the format is the same as for Fig. 8). In this case the reference system based on the minimum variance analysis is not appropriate because the spacecraft does not cross the main magnetopause current, but the parallel current sheets within the boundary layer, where the minimum variance analysis does not gives reliable results. Instead we use $\boldsymbol{N}=\boldsymbol{L} \times(\boldsymbol{n} \times \boldsymbol{L})$, where $\boldsymbol{n}$ is the boundary normal given by the boundary velocity $\boldsymbol{v}_{\text {dis }}=v_{\text {dis }} \boldsymbol{n}$ which is determined from the time delays between observations of the boundary by different spacecraft. The timing analysis is made on both the parallel current sheet and the density gradient to give the best fit over the boundary. Note that this parallel current sheet is not the main magnetopause current layer, but a local current sheet located on the magnetospheric side from the magnetopause current layer on the border between the magnetosphere (separatrix region) and the outflow region. The $L$ direction is the direction of the magnetic field inside the magnetosphere. $M$ completes the orthogonal system $M=N \times \boldsymbol{L}$.

In the event at 12:51 UT the longitudinal component of the magnetic field ( $B_{L}$ in Fig. 9a) decreases to almost zero but does not change sign. It means that the spacecraft does not cross the main magnetopause current layer but enters into it and then return back to the magnetosphere. At 12:51:46 UT perpendicular electrons in Fig. 9j show a characteristic transition from the hot magnetospheric plasma (electron temperature $T_{e} \sim 3 \mathrm{keV}$ ) to the colder magnetosheath-like plasma $\left(T_{e} \sim 80 \mathrm{eV}\right)$. Low energy perpendicular electrons are not present prior to the transition. The flux of high energy magnetospheric perpendicular electrons drastically decreases at the transition; however, some of these electrons are still present after the transition. The first magnetosheath ions (energies between $100 \mathrm{eV}-3 \mathrm{keV}$ ) are observed at the transition corresponding to the ion edge (purple bar), as seen in the high resolution time-energy ion spectrogram (Fig. 9f). Magnetospheric ions $(\sim 10 \mathrm{keV})$ are still present after the ion edge. After 12:51:46 UT there are mixed ions and electrons from the magnetosheath and the magnetosphere. At the same time the ion velocity (not shown) increases to about $300 \mathrm{~km} / \mathrm{s}$ which means that the spacecraft enters the ion jet (reconnection outflow/field reversal region). The density in the outflow region $\left(n \sim 6 \mathrm{~cm}^{-3}\right)$ is significantly higher than the magnetospheric density $\left(n \sim 0.5 \mathrm{~cm}^{-3}\right)$.

The region prior to 12:51:33 UT we identify as the separatrix (marked with a yellow bar in Fig. 9). The identification of this region as the separatrix is based on observing the electrons parallel to the magnetic field in Fig. 9k (at times when the parallel sector is aligned with $B$, i.e. the angle in Fig. 91 is close to zero). Parallel electrons are observed at energies 


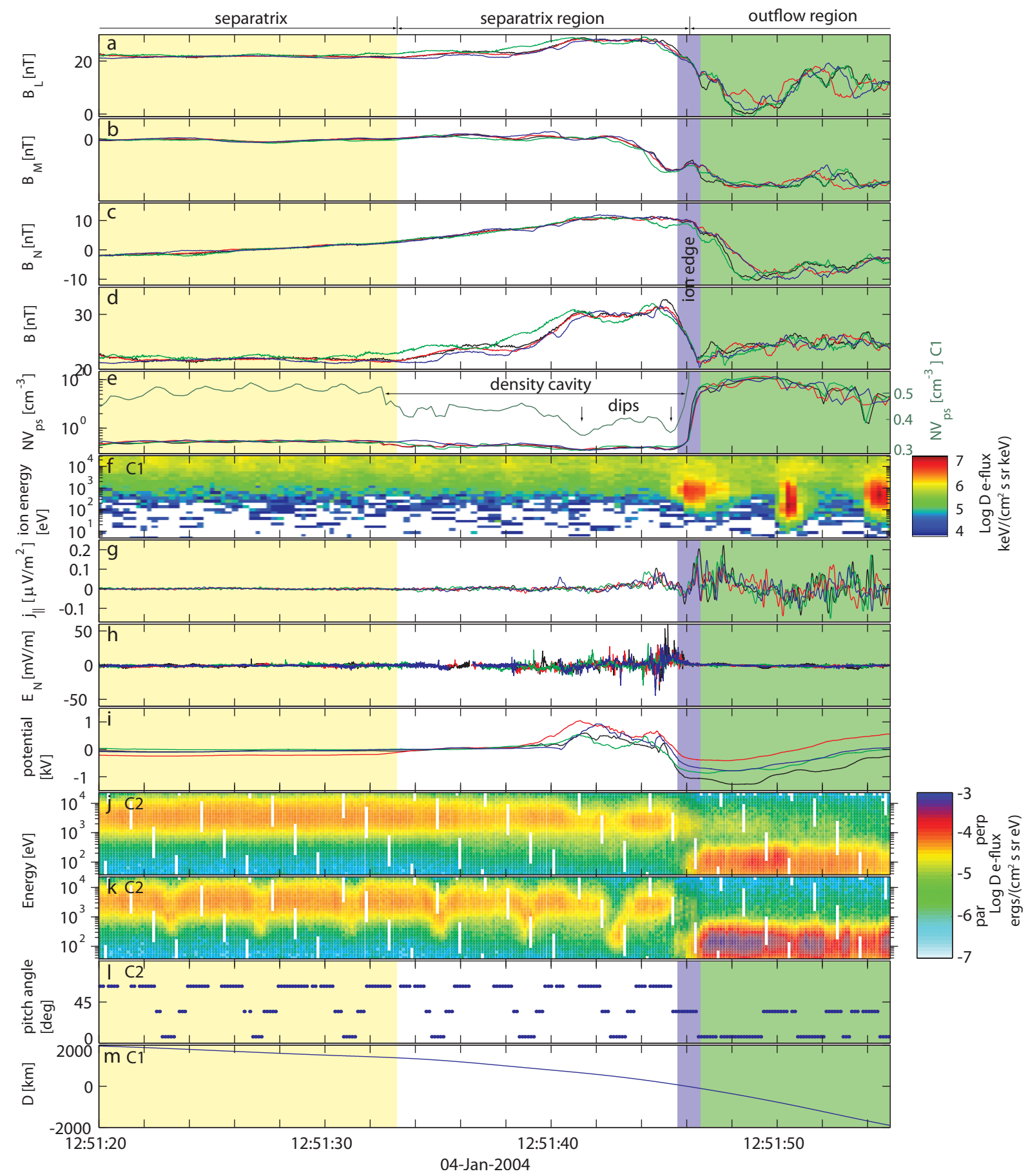

Fig. 9. Cluster crossing from the magnetosphere into the reconnection layer on 4 January 2004, 12:51 UT. Format of the figure is the same

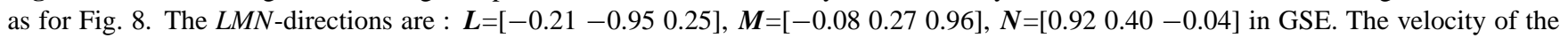
discontinuity is $\boldsymbol{v}_{\text {dis }}=176[-0.92-0.400 .04] \mathrm{km} / \mathrm{s}$ GSE. The time axes for spacecraft $1-4$ are shifted by $[-0.420-1.400 .03] \mathrm{s}$.

$\sim 0.5-1 \mathrm{keV}$ which is lower than the plasma sheet energies and, thus, originate from the magnetosheath, i.e. being the most energetic part of the magnetosheath population. We expect to observe such magnetosheath electrons only very close to the first opened field line. A similar distribution with parallel magnetosheath $\sim 1 \mathrm{keV}$ electrons is observed at the separatrix during the first event (see Fig. 8, 13:11:28 UT). In contrast to the first event, where such a distribution is observed 
only during one spacecraft spin and the separatrix can be located very precisely, such a distribution is observed for many spins in this second event and the separatrix is observed for a longer time interval in Fig. 9. However, this interval still corresponds to a very narrow region in space. For this event we calculate the distance in the same way as for the previous event. Prior to 12:51:33 UT the spacecraft move very slowly and efficiently stay at the same position relative to the magnetopause (Fig. 9m).

At 12:51:33 UT the normal velocity increases to $\sim 180 \mathrm{~km} / \mathrm{s}$ (corresponding to the slope of the distance $D$ in Fig. $9 \mathrm{~m}$ ) and the spacecraft cross the separatrix region reaching the ion edge at $\sim 12: 51: 46 \mathrm{UT}$. The width of the separatrix region is $\sim 2100 \mathrm{~km}$. Similar to the first event, the separatrix region coincides with the density cavity and the increased total magnetic field. As the spacecraft cross the separatrix region and get closer to the ion edge the characteristic energy of parallel electrons continuously decreases (Fig. 9k); the energy of the parallel electrons is changing more gradually than the rapid change in the perpendicular electrons at 12:51:46 UT in Fig. 9j. After the ion edge (12:51:47 UT) magnetosheath electrons are the dominant population.

Strong electric fields normal to the boundary $\left(E_{N}\right.$, Fig. $\left.9 \mathrm{~h}\right)$ with amplitude up to $50 \mathrm{mV} / \mathrm{m}$ are observed inside the separatrix region. By integrating the normal electric field we get the electric potential which will affect ions crossing the separatrix region (Fig. 9i). Inside the separatrix region the potential first increases and then decreases and in total the electric potential changes by $1.4 \mathrm{kV}$ across the region. The steady potential increase inside the outflow region (after 12:51:47 UT) is due to the large plasma convection there. The strongest electric fields are localized on the low density side of the main density gradient. In Fig. 9g there is a current parallel to the magnetic field with a magnitude of $0.2 \mu \mathrm{A} / \mathrm{m}^{2}$ at 12:51:47 UT. The parallel current is coinciding with the density gradient located on the ion edge (I2) in Fig. 1. The magnitude of this parallel current and its location is the same as for the first event. The calculation of the current depends on the timing analysis which is valid up to only 12:51:47 UT for this event so the current estimation is no longer valid.

\section{Discussion}

We have analyzed in detail eight crossings of the magnetopause reconnection layer (marked by vertical lines in Fig. 3) which are consistent with the spacecraft crossing separatrix regions of reconnection sites during ongoing reconnection. Evidence for ongoing reconnection are based on the properties of the observed reconnection ion jets and observations of FTEs as well as observations of separatrix regions. The Cooling model shows that the observations are consistent with a reconnection site located sunward of the spacecraft.
All the events we analyzed show a very similar boundary structure to the ones presented in detail in Fig. 8 and 9. Table 1 presents a summary of the main characteristics for all these events: the boundary velocity, the width of the separatrix region (distance between the separatrix and the main density gradient), potential variation inside the separatrix region and the maximum parallel current. For all of our events summarized in Table 1 we observe a density cavity between the magnetosphere and the outflow region (ion jet). The cavities contain a density decrease down to about half of the magnetospheric density and have a width ranging from $300 \mathrm{~km}\left(\sim 1 c / \omega_{p i(M S)} \sim 4 c / \omega_{p i(S H)}\right)$ to $2500 \mathrm{~km}$ $\left(\sim 9 c / \omega_{p i(M S)} \sim 34 c / \omega_{p i(S H)}\right)$. We find cavities which are on average much wider than the one found by André et al. (2004) that is about $300 \mathrm{~km}$, and by Retinò et al. (2006) which is only $100 \mathrm{~km}$ wide. A discussion on how the density cavity is created can be found in Khotyaintsev et al. (2006). We could not identify the cause of different cavity widths. Our typical values of the width of the separatrix region can be compared with the separatrix region width estimates at much lower altitude in the mid altitude cusp region by Bogdanova et al. (2006). They find the separatrix region to have widths up to $2^{\circ}$ ILAT with $0.2^{\circ}$ ILAT being characteristic value. This width corresponds to a width of $\sim 600 \mathrm{~km}$ near the magnetopause when mapped along the magnetic field and thus falls within the range of our estimates.

Almost all the magnetopause reconnection layer crossings show similar characteristic properties of the separatrix region. The main density gradient defines the edge of the ion jet and the ion boundary to the the separatrix region. The outflow region is dominated by plasma of magnetosheath origin. In the data we see that the outflow region and the magnetopause current layer, identified as the time interval when the $B_{L}$-component rotate from positive to negative values, at least sometimes overlap (see Fig. 7 and Fig. 8). When the spacecraft is crossing from the magnetosheath to the magnetosphere, there is a sharp change in the ion distribution at the ion edge; the magnetospheric population becomes dominant, and a rather sharp change in the perpendicular electrons is observed. The parallel electrons, on the other hand, are changing more gradually and represent a mixture of the magnetosheath and magnetospheric populations throughout the separatrix region. Observations of this mixing of magnetosheath and magnetospheric electron populations inside the separatrix region provide additional (kinetic) evidence for ongoing reconnection. Closer to the main density gradient we observe mostly the low energy magnetosheath electrons transmitted through the magnetopause. The flux of such electrons decreases as we move deeper into the magnetosphere. Further away from the magnetopause in the magnetosphere we observe a loss cone distribution in high energy magnetospheric electrons created due to escape of energetic electrons with small pitch angles along the open field lines. At lower energies magnetosheath electrons are entering along the opened field lines. The magnetospheric edge 
Table 1. Summary of parameters of the observed separatrix regions on 4 January 2004. The table shows (1) the time of the event, (2) the velocity of the discontinuity, (3) the width of the separatrix region, (4) the potential change over the separatrix region with the starting point on the magnetospheric side of the separatrix region, (5) the potential dip with respect to the magnetospheric level and positive sign corresponds to diverging electric fields and (6) the magnitude of the parallel current. The potential dip is a potential change over a region smaller than the width of the separatrix region.

\begin{tabular}{cccccc}
\hline Time & $\begin{array}{c}v_{\text {dis }} \\
(\mathrm{km} / \mathrm{h})\end{array}$ & $\begin{array}{c}\text { width } \\
(\mathrm{km})\end{array}$ & $\begin{array}{c}\text { potential change } \\
(\mathrm{kV})\end{array}$ & $\begin{array}{c}\text { potential dip } \\
(\mathrm{kV})\end{array}$ & $\begin{array}{c}\text { current } \\
\left(\mu \mathrm{A} / \mathrm{m}^{2}\right)\end{array}$ \\
\hline $12: 47$ & 270 & 300 & $-2.6 \pm 1.3$ & - & 0.3 \\
$12: 51$ & 180 & 1400 & $-0.7 \pm 0.3$ & $0.8 \pm 0.3$ & 0.2 \\
$12: 58$ & 250 & 1500 & $1.1 \pm 0.6$ & $-1.0 \pm 0.4$ & 0.5 \\
$13: 06$ & 60 & 1500 & $5.4 \pm 0.9$ & - & 0.6 \\
$13: 09$ & 150 & 1500 & $5.4 \pm 1.6$ & - & 0.3 \\
$13: 11$ & 210 & 2300 & $3.8 \pm 0.7$ & - & 0.2 \\
$13: 46$ & 180 & 2500 & $0.8 \pm 0.8$ & $-1.5 \pm 0.5$ & 0.1 \\
$13: 55$ & 140 & 300 & $0.75 \pm 0.3$ & - & 0.2 \\
\hline
\end{tabular}

of the separatrix region is the separatrix, a transition from the open to closed magnetospheric field lines. Low energy (below the typical plasma sheet energies) field aligned electrons are no longer observed on the magnetospheric side of the separatrix. This point also coincides with the magnetospheric edge of the density cavity.

On the main density gradient which is located on the ion boundary of the magnetospheric separatrix region we observe a thin parallel current sheet with typical current densities between $0.1 \mu \mathrm{A} / \mathrm{m}^{2}$ and $0.6 \mu \mathrm{A} / \mathrm{m}^{2}$. Presumably, these field-aligned currents are at one end closed by the Hall currents in the ion diffusion region (Vaivads et al., 2004b), and at the other end most probably closed through the currents in the ionosphere. Mapping the field aligned current densities to the ionosphere results in expected field aligned current densities at the top of the ionosphere of $200 \mu \mathrm{A} / \mathrm{m}^{2}-1200 \mu \mathrm{A} / \mathrm{m}^{2}$. For the mapping we use that $B \sim 1 / R^{2}$ and $J \sim 1 / R^{2}$ so $B \sim J$ and a typical magnetic field value of $20 \mathrm{nT}$ in the magnetosphere and $4 \times 10^{4} \mathrm{nT}$ in the ionosphere. Large field aligned currents of hundreds $\mu \mathrm{A} / \mathrm{m}^{2}$ have previously been observed by the low altitude satellites such as Freja and Astrid-II on similar magnetospheric flux tubes (Stasiewicz et al., 1998; Ivchenko and Marklund, 2002). In the ionosphere the manifestation of field-aligned currents originating in the magnetosheath have been observed, e.g. in the form of poleward moving auroral forms (PMAFs) (Sandholt et al., 1998).

All observed separatrix regions are associated with strong electric fields. The strongest electric field component is in the direction normal to the boundary and the values that we have observed (e.g. in Fig. 8 and Fig. 9) are of the same magnitude as previously observed by André et al. (2004) and Retinò et al. (2006). Note that it is the tangential electric field and not the normal electric field that corresponds to the plasma motion across the boundary. The normal electric field has a fast fluctuating component with frequency above a few $\mathrm{Hz}$, that is most probably related to electrostatic lower hybrid drift waves that propagate along the cavity (Vaivads et al., 2004a). The normal electric field can be integrated to obtain the electric potential structure across the separatrix region. The studied separatrix regions show that there exists a potential jump across the separatrix region varying between $0.3 \mathrm{kV}$ and $5.5 \mathrm{kV}$ (Table 1). The electric fields observed locally close to the magnetopause will map along the magnetic field lines into the ionosphere. Thus the local potential drops that are observed across the separatrix region at the magnetopause can be expected to lead to similar potential drops down in the ionosphere. The observed values are of the same magnitude as the ones observed across the dayside auroral region at low altitudes supporting the idea that such potential jumps can map down into the ionosphere (Lundin et al., 1995). The shape of the electric potential across the separatrix regions is different for the different events; for example, in the event of 12:51 in Fig. 9i the electric potential first increased and then decreased, which corresponds to diverging electric fields, i.e. pointing outwards from the separatrix region. For another event at 13:11 in Fig. 8i the potential monotonically decreases over the separatrix region and the corresponding electric field is directed from the magnetosphere to the magnetosheath, similar to the event of Khotyaintsev et al. (2006). It is important to notice that when the normal electric field extension in the normal direction is sufficiently small (in comparison to the ion gyro-radius) ions entering the separatrix region are accelerated by the normal electric field and in this way obtain energy comparable to the potential jump within the separatrix region. This can be particularly important for cold ionospheric ions that in this way can increase their energy hundredfold. There are no ionospheric ions detected for the current event, however, preliminary studies of other events show that energization of ionospheric ions within the separatrix region can be significant. Also Topliss et al. (2001), Bogdanova et al. (2004) and Bogdanova et al. (2006) observed energized ionospheric ions in 


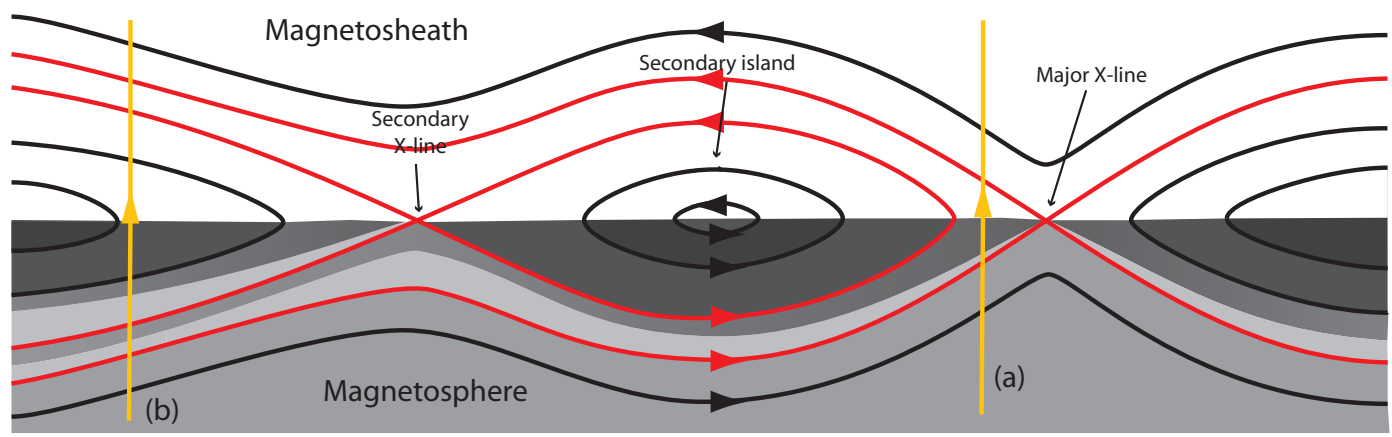

Fig. 10. Illustration of the spacecraft crossings of the magnetopause during reconnection due to multiple $X$-lines. The major $X$-line is on the right and the secondary X-line is on the left. Separatrices associated with the X-lines are marked in red and plasma density is shaded in grey, density cavities associated with the X-lines can be seen as light grey bands next to the separatrices. Two example crossings by spacecraft are marked in yellow. The spacecraft on the left crosses a wide cavity with multiple dips due to multiple X-lines, the spacecraft on the right crosses closer to the major X-line and sees only one narrow cavity.

the separatrix region. Earlier studies show that the strong normal electric fields are mainly Hall fields, $\boldsymbol{E} \sim \boldsymbol{j} \times \boldsymbol{B} / n e$, and do not correspond to the large scale motion of the plasma (Khotyaintsev et al., 2006).

In 7 out of 8 events we observe a complex internal structure of the separatrix region with multiple density dips inside the cavities. The origin of the dips is not fully understood. One possibility involves existence of multiple X-lines (and multiple separatrices) due to formation of secondary magnetic islands at the reconnection site. The existence of such islands has been confirmed both using numerical simulations (Daughton et al., 2006) and spacecraft observations (Eastwood et al., 2007; Chen et al., 2008). If the formation of a density cavity is characteristic for every active $\mathrm{X}$-line we could use this to monitor whether reconnection is ongoing at a single or multiple X-lines. Figure 10 illustrates spacecraft crossings of the magnetopause with two X-lines. The yellow line shows the spacecraft orbit. In the crossing (a) to the right in Fig. 10 the spacecraft is crossing the magnetopause near the major X-line and only observe a single separatrix on the magnetospheric side. The density in Fig. 10 is illustrated by the grey background color. Light grey color corresponds to low density and darker to high density. The single separatrix crossing then corresponds to the crossing of a single density cavity. In the crossing (b) to the left in Fig. 10 there is another X-line present between the spacecraft and the major X-line. In this case we observe a density cavity with two density dips corresponding to crossing two separatrices, one from the major X-line and one from the secondary X-line. In the future it is necessary to test if similar wide density cavities with multiple dips can be produced in numerical simulations and whether multiple dips are correlated with the presence of multiple $\mathrm{X}$-lines.

\section{Conclusions}

We have studied Cluster spacecraft observations in the vicinity of the dayside magnetopause on 4 January 2004, between 12:30 and 14:30 UT. During this time the IMF was varying but mainly directed southward and we find signatures of ongoing reconnection. We analyze in detail eight encounters of the separatrix region of magnetic reconnection on the magnetospheric side of the magnetopause current layer, i.e. the region located between the inner (magnetospheric) separatrix and the reconnection jet.

We find a similar structure of the separatrix region in all our events: (1) a density cavity, (2) strong parallel currents which coincide with the ion boundary, (3) strong electric fields and (4) large potential variations across the region. In most events there are multiple density dips inside the cavities. The total width of the cavities varies between $300 \mathrm{~km}$ and $2500 \mathrm{~km}$ which is from a few to a few tens of the ion inertial length and consistent with observations at much lower altitudes (Bogdanova et al., 2006). The electron distributions observed inside the separatrix regions show a gradual change in the parallel energy due to transition from the magnetospheric to magnetosheath electron population that is consistent with expected signatures when magnetospheric and magnetosheath electrons mix on newly opened field lines.

The strong parallel currents are concentrated on the main density gradient on the magnetosheath side of the cavity. Only in one earlier case study by André et al. (2004) the strong parallel current has been found to be located on the magnetospheric side of the cavity. The observed strong currents on the ion boundary of the separatrix region, when mapped into the ionosphere would correspond to very strong ionospheric currents, more than $200 \mu \mathrm{A} / \mathrm{m}^{2}$, which can be important for formation of the aurora and global magnetosphere-ionosphere coupling. 
Inside the density cavity we find strong electric fields directed normal to the boundary. We integrate this electric field to obtain the electric potential structure across the separatrix region. The electric potential shows potential jumps of several $\mathrm{kV}$ across the separatrix region. In some cases there can be internal structure of the electric potential showing diverging electric fields but in most cases the potential changes monotonically across the separatrix region. The strong electric fields within the separatrix region can contribute to the energization (by a few $\mathrm{keV}$ ), of the ions crossing this region. This can be particularly important for cold ionospheric ions.

Acknowledgements. We Would like to thank CIS, FGM and PEACE teams as well as the ESA Cluster Active Archive for providing the data.

Topical Editor I. A. Daglis thanks two anonymous referees for their help in evaluating this paper.

\section{References}

André, M., Vaivads, A., Buchert, S. C., Fazakerley, A. N., and Lahiff, A.: Thin electron-scale layers at the magnetopause, Geophys. Res. Lett., 31, 3803, doi:10.1029/2003GL018137, 2004.

Balogh, A., Carr, C. M., Acuña, M. H., Dunlop, M. W., Beek, T. J., Brown, P., Fornacon, K.-H., Georgescu, E., Glassmeier, K.H., Harris, J., Musmann, G., Oddy, T., and Schwingenschuh, K.: The Cluster Magnetic Field Investigation: overview of in-flight performance and initial results, Ann. Geophys., 19, 1207-1217, 2001 , http://www.ann-geophys.net/19/1207/2001/.

Biernat, H. K., Semenov, V. S., Drobysh, O. A., and Heyn, M. F.: Magnetic reconnection: Observations on October 29, 1979, and model results, J. Geophys. Res., 103, 11919-11928, doi: 10.1029/98JA00587, 1998.

Biskamp, D.: Magnetic reconnection in Plasmas, Cambridge University Press, iSBN 052158288 1, 2000.

Bogdanova, Y. V., Fazakerley, A. N., Owen, C. J., Klecker, B., Cornilleau-Wehrlin, N., Grison, B., André, M., Cargill, P., Rème, H., Bosqued, J. M., Kistler, L. M., and Balogh, A.: Correlation between suprathermal electron bursts, broadband extremely low frequency waves, and local ion heating in the midaltitude cleft/low-latitude boundary layer observed by Cluster, J. Geophys. Res. (Space Physics), 109, 12226, doi:10.1029/ 2004JA010554, 2004.

Bogdanova, Y. V., Owen, C. J., Fazakerley, A. N., Klecker, B., and Rème, H.: Statistical study of the location and size of the electron edge of the Low-Latitude Boundary Layer as observed by Cluster at mid-altitudes, Ann. Geophys., 24, 2645-2665, 2006, http://www.ann-geophys.net/24/2645/2006/.

Cattell, C., Dombeck, J., Wygant, J., Drake, J. F., Swisdak, M., Goldstein, M. L., Keith, W., Fazakerley, A., André, M., Lucek, E., and Balogh, A.: Cluster observations of electron holes in association with magnetotail reconnection and comparison to simulations, J. Geophys. Res. (Space Physics), 110, 1211, doi: 10.1029/2004JA010519, 2005.

Chen, L.-J., Bhattacharjee, A., Puhl-Quinn, P. A., Yang, H., Bessho, N., Imada, S., Mühlbachler, S., Daly, P. W., Lefebvre, B., Khotyaintsev, Y., Vaivads, A., Fazakerley, A., and Georgescu,
E.: Observation of energetic electrons within magnetic islands, Nature Physics, 4, 19-23, doi:10.1038/nphys777, 2008.

Cooling, B. M. A., Owen, C. J., and Schwartz, S. J.: Role of the magnetosheath flow in determining the motion of open flux tubes, J. Geophys. Res., 106, 18763-18776, doi:10.1029/ 2000JA000455, 2001.

Crooker, N. U.: Dayside merging and cusp geometry, J. Geophys. Res., 84, 951-959, doi:10.1029/JA084iA03p00951, 1979.

Daughton, W., Scudder, J., and Karimabadi, H.: Fully kinetic simulations of undriven magnetic reconnection with open boundary conditions, Phys. Plasmas, 13, 2101, doi:10.1063/1.2218817, 2006.

Eastwood, J. P., Phan, T.-D., Mozer, F. S., Shay, M. A., Fujimoto, M., Retinò, A., Hesse, M., Balogh, A., Lucek, E. A., and Dandouras, I.: Multi-point observations of the Hall electromagnetic field and secondary island formation during magnetic reconnection, J. Geophys. Res. (Space Physics), 112, 6235, doi: 10.1029/2006JA012158, 2007.

Escoubet, C. P., Pedersen, A., Schmidt, R., and Lindqvist, P. A.: Density in the magnetosphere inferred from ISEE 1 spacecraft potential, J. Geophys. Res., 102, 17595-17610, doi:10.1029/ 97JA00290, 1997.

Gosling, J. T., Thomsen, M. F., Bame, S. J., Onsager, T. G., and Russell, C. T.: The electron edge of the low latitude boundary layer during accelerated flow events, Geophys. Res. Lett., 17, 1833-1836, 1990.

Gustafsson, G., André, M., Carozzi, T., Eriksson, A. I., Fälthammar, C.-G., Grard, R., Holmgren, G., Holtet, J. A., Ivchenko, N., Karlsson, T., Khotyaintsev, Y., Klimov, S., Laakso, H., Lindqvist, P.-A., Lybekk, B., Marklund, G., Mozer, F., Mursula, K., Pedersen, A., Popielawska, B., Savin, S., Stasiewicz, K., Tanskanen, P., Vaivads, A., and Wahlund, J.-E.: First results of electric field and density observations by Cluster EFW based on initial months of operation, Ann. Geophys., 19, 1219-1240, 2001, http://www.ann-geophys.net/19/1219/2001/.

Haaland, S., Paschmann, G., and Sonnerup, B. U. Ö.: Comment on "A new interpretation of Weimer et al.'s solar wind propagation delay technique" by Bargatze et al., J. Geophys. Res. (Space Physics), 111, 6102, doi:10.1029/2005JA011376, 2006.

Ivchenko, N. and Marklund, G.: "Current singularities" observed on Astrid-2, Adv. Space Res., 30, 1779-1782, 2002.

Johnstone, A. D., Alsop, C., Burge, S., Carter, P. J., Coates, A. J., Coker, A. J., Fazakerley, A. N., Grande, M., Gowen, R. A., Gurgiolo, C., Hancock, B. K., Narheim, B., Preece, A., Sheather, P. H., Winningham, J. D., and Woodliffe, R. D.: Peace: a Plasma Electron and Current Experiment, Space Sci. Rev., 79, 351-398, doi:10.1023/A:1004938001388, 1997.

Khotyaintsev, Y., Buchert, S., Stasiewicz, K., Vaivads, A., Savin, S., Papitashvili, V. O., Farrugia, C. J., Popielawska, B., and Tung, Y.-K.: Transient reconnection in the cusp during strongly negative IMF By, J. Geophys. Res. (Space Physics), 109, 4204, doi: 10.1029/2003JA009908, 2004.

Khotyaintsev, Y. V., Vaivads, A., Retinò, A., André, M., Owen, C. J., and Nilsson, H.: Formation of Inner Structure of a Reconnection Separatrix Region, Phys. Rev. Lett., 97, 205003, doi: 10.1103/PhysRevLett.97.205003, 2006.

Kobel, E. and Fluckiger, E. O.: A model of the steady state magnetic field in the magnetosheath, J. Geophys. Res., 99, 23617, 
doi:10.1029/94JA01778, 1994

Levy, R. H., Petschek, H. E., and Siscoe, G. L.: Aerodynamic aspects of the magnetospheric flow, AIAA, 2, 2065-2076, 1964.

Lin, Y. and Lee, L. C.: Reconnection layer at the flank magnetopause in the presence of shear flow, Geophys. Res. Lett., 21, 855-858, doi:10.1029/94GL00704, 1994.

Lockwood, M., Onsager, T. G., Davis, C. J., Smith, M. F., and Denig, W. F.: The characteristic of the magnetopause reconnection X-line deduced from low-altitude satellite observations of cusp ions, Geophys. Res. Lett., 21, 2757-2760, doi:10.1029/ 94GL02696, 1994.

Lockwood, M., Cowley, S. W. H., and Onsager, T. G.: Ion acceleration at both the interior and exterior Alfvén waves associated with the magnetopause reconnection site: Signatures in cusp precipitation, J. Geophys. Res., 101, 21501-21514, doi: 10.1029/96JA01948, 1996.

Luhr, H., Warnecke, J. F., and Rother, M. K. A.: An algorithm for estimating field-aligned currents from single spacecraft magnetic field measurements: a diagnostic tool applied to Freja satellite data, IEEE Transactions on Geoscience and Remote Sensing, 34, 1369-1376, doi:10.1109/36.544560, 1996.

Lundin, R., Yamauchi, M., Woch, J., and Marklund, G.: Boundary layer polarization and voltage in the 14 MLT region, J. Geophys. Res., 100, 7587-7597, 1995.

McComas, D. J., Bame, S. J., Barker, P., Feldman, W. C., Phillips, J. L., Riley, P., and Griffee, J. W.: Solar Wind Electron Proton Alpha Monitor (SWEPAM) for the Advanced Composition Explorer, Space Sci. Rev., 86, 563-612, doi:10.1023/A: 1005040232597, 1998.

Mozer, F. S., Bale, S. D., and Phan, T. D.: Evidence of Diffusion Regions at a Subsolar Magnetopause Crossing, Phys. Rev. Lett., 89, 015002, doi:10.1103/PhysRevLett.89.015002, 2002.

Owen, C. J., Fazakerley, A. N., Carter, P. J., Coates, A. J., Krauklis, I. C., Szita, S., Taylor, M. G. G. T., Travnicek, P., Watson, G., Wilson, R. J., Balogh, A., and Dunlop, M. W.: Cluster PEACE observations of electrons during magnetospheric flux transfer events, Ann. Geophys., 19, 1509-1522, 2001, http://www.ann-geophys.net/19/1509/2001/.

Owen, C. J., Marchaudon, A., Dunlop, M. W., Fazakerley, A. N., Bosqued, J.-M., Dewhurst, J. P., Fear, R. C., Fuselier, S. A., Balogh, A., and Rème, H.: Cluster observations of "crater" flux transfer events at the dayside high-latitude magnetopause, J. Geophys. Res. (Space Physics), 113, 7, doi:10.1029/ 2007JA012701, 2008.

Pedersen, A., Lybekk, B., André, M., Eriksson, A., Masson, A., Mozer, F. S., Lindqvist, P.-A., Décréau, P. M. E., Dandouras, I., Sauvaud, J.-A., Fazakerley, A., Taylor, M., Paschmann, G., Svenes, K. R., Torkar, K., and Whipple, E.: Electron density estimations derived from spacecraft potential measurements on Cluster in tenuous plasma regions, J. Geophys. Res. (Space Physics), 113, 7, doi:10.1029/2007JA012636, 2008.

Petschek, H. E.: Magnetic Field Annihilation, pp. 425-+, 1964.

Pritchett, P. L. and Coroniti, F. V.: Three-dimensional collisionless magnetic reconnection in the presence of a guide field, J. Geophys. Res. (Space Physics), 109, 1220, doi:10.1029/ 2003JA009999, 2004.

Rème, H., Aoustin, C., Bosqued, J. M., Dandouras, I., Lavraud, B., Sauvaud, J. A., Barthe, A., Bouyssou, J., Camus, Th., CoeurJoly, O., Cros, A., Cuvilo, J., Ducay, F., Garbarowitz, Y., Medale,
J. L., Penou, E., Perrier, H., Romefort, D., Rouzaud, J., Vallat, C., Alcaydé, D., Jacquey, C., Mazelle, C., d'Uston, C., Möbius, E., Kistler, L. M., Crocker, K., Granoff, M., Mouikis, C., Popecki, M., Vosbury, M., Klecker, B., Hovestadt, D., Kucharek, H., Kuenneth, E., Paschmann, G., Scholer, M., Sckopke, N., Seidenschwang, E., Carlson, C. W., Curtis, D. W., Ingraham, C., Lin, R. P., McFadden, J. P., Parks, G. K., Phan, T., Formisano, V., Amata, E., Bavassano-Cattaneo, M. B., Baldetti, P., Bruno, R., Chionchio, G., Di Lellis, A., Marcucci, M. F., Pallocchia, G., Korth, A., Daly, P. W., Graeve, B., Rosenbauer, H., Vasyliunas, V., McCarthy, M., Wilber, M., Eliasson, L., Lundin, R., Olsen, S., Shelley, E. G., Fuselier, S., Ghielmetti, A. G., Lennartsson, W., Escoubet, C. P., Balsiger, H., Friedel, R., Cao, J.-B., Kovrazhkin, R. A., Papamastorakis, I., Pellat, R., Scudder, J., and Sonnerup, B.: First multispacecraft ion measurements in and near the Earth's magnetosphere with the identical Cluster ion spectrometry (CIS) experiment, Ann. Geophys., 19, 1303-1354, 2001, http://www.ann-geophys.net/19/1303/2001/.

Retinò, A., Vaivads, A., André, M., Sahraoui, F., Khotyaintsev, Y., Pickett, J. S., Bavassano Cattaneo, M. B., Marcucci, M. F., Morooka, M., Owen, C. J., Buchert, S. C., and Cornilleau-Wehrlin, N.: Structure of the separatrix region close to a magnetic reconnection X-line: Cluster observations, Geophys. Res. Lett., 33, 6101, doi:10.1029/2005GL024650, 2006.

Robert, P., Dunlop, M. W., Roux, A., and Chanteur, G.: Accuracy of Current Density Determination, in: Analysis Methods for Multi-Spacecraft Data, edited by Paschmann, G. and Daly, P. W., chap. 16, pp. 395-418, Int. Space Sci. Inst., Bern., 2000.

Russell, C. T. and Elphic, R. C.: Initial ISEE magnetometer results - Magnetopause observations, Space Sci. Rev., 22, 681-715, doi: 10.1007/BF00212619, 1978.

Sandholt, P. E., Farrugia, C. J., Moen, J., Noraberg, Ø., Lybekk, B., Sten, T., and Hansen, T.: A classification of dayside auroral forms and activities as a function of interplanetary magnetic field orientation, J. Geophys. Res., 103, 23325-23346, doi:10.1029/ 98JA02156, 1998.

Sandholt, P. E., Denig, W. F., Farrugia, C. J., Lybekk, B., and Trondsen, E.: Auroral structure at the cusp equatorward boundary: Relationship with the electron edge of low-latitude boundary layer precipitation, J. Geophys. Res. (Space Physics), 107, 1235, doi: 10.1029/2001JA005081, 2002.

Scholer, M.: Models of Flux Transfer Events, pp. 235, Physics of the Magnetopause, 1995.

Schwartz, S. J.: Shock and Discontinuity Normals, Mach Numbers, and Related Parameters, in: Analysis Methods for MultiSpacecraft Data, edited by: Paschmann, G. and Daly, P. W., chap. 10, pp. 249-270, Int. Space Sci. Inst., Bern., 2000.

Semenov, V. S., Kubyshkin, I. V., Lebedeva, V. V., Rijnbeek, R. P., Heyn, M. F., Biernat, H. K., and Farrugia, C. J.: A comparison and review of steady-state and time-varying reconnection, Planet. Space Sci., 40, 63-87, doi:10.1016/0032-0633(92)90150-M, 1992.

Shay, M. A., Drake, J. F., Rogers, B. N., and Denton, R. E.: Alfvénic collisionless magnetic reconnection and the Hall term, J. Geophys. Res., 106, 3759-3772, doi:10.1029/1999JA001007, 2001.

Shue, J.-H., Chao, J. K., Fu, H. C., Russell, C. T., Song, P., Khurana, K. K., and Singer, H. J.: A new functional form to study the solar wind control of the magnetopause size and shape, J. Geophys. 
Res., 102, 9497-9512, doi:10.1029/97JA00196, 1997.

Smith, C. W., L'Heureux, J., Ness, N. F., Acuña, M. H., Burlaga, L. F., and Scheifele, J.: The ACE Magnetic Fields Experiment, Space Sci. Rev., 86, 613-632, doi:10.1023/A:1005092216668, 1998.

Sonnerup, B. U. O., Paschmann, G., Papamastorakis, I., Sckopke, N., Haerendel, G., Bame, S. J., Asbridge, J. R., Gosling, J. T., and Russell, C. T.: Evidence for magnetic field reconnection at the earth's magnetopause, J. Geophys. Res., 86, 10049-10067, 1981.

Stasiewicz, K., Holmgren, G., and Zanetti, L.: Density depletions and current singularities observed by Freja, J. Geophys. Res., 103, 4251-4260, doi:10.1029/97JA02007, 1998.

Topliss, S., Johnstone, A., Coates, A., Peterson, W. K., Kletzing, C. A., and Russell, C. T.: Charge neutrality and ion conic distributions at the equatorward electron edge of the midaltitude cusp, J. Geophys. Res., 106, 21095-21108, doi:10.1029/ 2000JA003032, 2001.

Tsyganenko, N. A.: Modeling the Earth's magnetospheric magnetic field confined within a realistic magnetopause, J. Geophys. Res., 100, 5599-5612, doi:10.1029/94JA03193, 1995.

Vaivads, A., André, M., Buchert, S. C., Wahlund, J.-E., Fazakerley, A. N., and Cornilleau-Wehrlin, N.: Cluster observations of lower hybrid turbulence within thin layers at the magnetopause, Geophys. Res. Lett., 31, 3804, doi:10.1029/2003GL018142, 2004a.
Vaivads, A., Khotyaintsev, Y., André, M., Retinò, A., Buchert, S. C., Rogers, B. N., Décréau, P., Paschmann, G., and Phan, T.: Structure of the Magnetic Reconnection Diffusion Region from Four-Spacecraft Observations, Phys. Rev. Lett., 93, 105001, doi: 10.1103/PhysRevLett.93.105001, 2004b.

Vaivads, A., Retinò, A., and André, M.: Microphysics of Magnetic Reconnection, Space Sci. Rev., 122, 19-27, doi:10.1007/ s11214-006-7019-3, 2006.

Weimer, D. R., Ober, D. M., Maynard, N. C., Collier, M. R., McComas, D. J., Ness, N. F., Smith, C. W., and Watermann, J.: Predicting interplanetary magnetic field (IMF) propagation delay times using the minimum variance technique, J. Geophys. Res. (Space Physics), 108, 1026, doi:10.1029/2002JA009405, 2003.

Wygant, J. R., Cattell, C. A., Lysak, R., Song, Y., Dombeck, J., McFadden, J., Mozer, F. S., Carlson, C. W., Parks, G., Lucek, E. A., Balogh, A., Andre, M., Reme, H., Hesse, M., and Mouikis, C.: Cluster observations of an intense normal component of the electric field at a thin reconnecting current sheet in the tail and its role in the shock-like acceleration of the ion fluid into the separatrix region, J. Geophys. Res. (Space Physics), 110, 9206, doi:10.1029/2004JA010708, 2005. 